\title{
A miRNA-200c/cathepsin L feedback loop determines paclitaxel resistance in human lung cancer A549 cells in vitro through regulating epithelial-mesenchymal transition
}

\author{
Yi-fan ZHAO ${ }^{1, \#}$, Mei-ling $\mathrm{HAN}^{1,2, \#}$, Ya-jie XIONG ${ }^{1}$, Long WANG ${ }^{1}$, Yao FEI ${ }^{1}$, Xiao SHEN ${ }^{1}$, Ying ZHU ${ }^{1}$, Zhong-qin LIANG ${ }^{1, *}$ \\ ${ }^{1}$ Department of Pharmacology, College of Pharmaceutical Sciences, Soochow University, Suzhou 215123, China; ${ }^{2}$ Department of \\ Pharmacy, Wuxi Children's Hospital, Wuxi 214013, China
}

\begin{abstract}
Cathepsin L (CTSL), a cysteine protease, is closely related to tumor occurrence, development, and metastasis, and possibly regulates cancer cell resistance to chemotherapy. miRNAs, especially the miR-200 family, have been implicated in drug-resistant tumors. In this study we explored the relationship of CTSL, miRNA-200c and drug resistance, and the potential regulatory mechanisms in human lung cancer A549 cells and A549/TAX cells in vitro. A549/TAX cells were paclitaxel-resistant A549 cells overexpressing CTSL and characterized by epithelial-mesenchymal transition (EMT). We showed that miRNA-200c and CTSL were reciprocally linked in a feedback loop in these cancer cells. Overexpression of miRNA-200c in A549/TAX cells decreased the expression of CTSL, and enhanced their sensitivity to paclitaxel and suppressed EMT, whereas knockdown of miRNA-200c in A549 cells significantly increased the expression of CTSL, and decreased their sensitivity to paclitaxel and induced EMT. Overexpression of CTSL in A549 cells significantly decreased the expression of miRNA-200c, and reduced their sensitivity to paclitaxel and induced EMT, but these effects were reversed by miRNA-200c, whereas knockdown of CTSL in A549/TAX cells attenuated paclitaxel resistance and remarkably inhibited EMT, but the inhibition of miRNA-200c could reverse these effects. Therefore, miRNA-200c may be involved in regulating paclitaxel resistance through CTSL-mediated EMT in A549 cells, and CTSL and miRNA-200c are reciprocally linked in a feedback loop.
\end{abstract}

Keywords: human lung cancer; paclitaxel resistance; CTSL; miRNA-200c; EMT; feedback loop

Acta Pharmacologica Sinica (2018) 39: 1034-1047; doi: 10.1038/aps.2017.164; published online 7 Dec 2017

\section{Introduction}

The morbidity of lung cancer is currently the highest among the morbidities of all cancer types ${ }^{[1]}$, and this disease is mainly treated with chemotherapy ${ }^{[2,3]}$. However, the application of chemotherapy is limited by therapeutic failure because of treatment resistance ${ }^{[4]}$. Thus, the mechanism of drug resistance should be elucidated and effective targets should be developed for lung cancer treatment.

Cathepsin L (CTSL) is a cysteine protease belonging to the papain-like family of cysteine proteinases, and this protease is closely related to tumor occurrence, development, and metastasis $^{[5-7]}$. CTSL plays an important role in the growth, survival, cycle, migration, and invasion of tumor cells ${ }^{[8]}$ and possibly

\footnotetext{
\# These authors contributed equally to this work.

* To whom correspondence should be addressed.

E-mail liangzhongqin@suda.edu.cn

Received 2017-07-03 Accepted 2017-09-18
}

regulates cancer cell resistance to chemotherapy. Zheng et al suggested that CTSL inhibition in drug-resistant cells facilitates the induction of senescence and the reversal of drug resistance $^{[9]}$, and CTSL inhibition-mediated drug target stabilization may be an alternative approach to enhance chemotherapeutic efficacy ${ }^{[10]}$. In our laboratory, CTSL is initially identified as a novel epithelial mesenchymal transition (EMT) regulator, and this protease is involved in the modulation of tumor cell invasion and migration ${ }^{[11]}$. Then we indicated that CTSL also regulates drug resistance by mediating EMT through its effects on the expression of EMT-associated transcription factors, Snail, Slug, ZEB1, and ZEB2 ${ }^{[12]}$. The overexpression of CTSL is associated with the chemoresistance and invasion of epithelial ovarian cancer ${ }^{[13]}$ and is related to gefitinib resistance in lung cancer ${ }^{[14]}$. Increased CTSL levels are associated with the increased chemoresistance of tumor cells and may be applied to gene therapy ${ }^{[15]}$. Thus, CTSL-mediated EMT may serve as a target to improve the efficacy of chemotherapeutics against 
lung cancer and other types of malignancies.

MicroRNAs (miRNAs) are small non-protein coding RNAs that modulate important cellular functions through their post-transcriptional regulation of messenger RNAs (mRNAs). miRNAs participate in the regulation of cell differentiation, growth, and death in normal and malignant tissues ${ }^{[16]}$. The role of miRNAs, especially the miR-200 family, is a crucial research field in drug-resistant tumors. The miRNA-200c family regulates EMT, which is implicated in cancer cell invasion and metastasis and in the drug resistance of many cancer types ${ }^{[17-27]}$. As a member of the miRNA-200 family, miRNA-200c functions as a key regulator of EMT in numerous cancers and promotes an epithelial phenotype by inhibiting several EMT genes, including ZEB1 and ZEB2 ${ }^{[28]}$. miRNA-200c expression can regulate EMT in bladder cancer cells and reverse their resistance to epidermal growth factor receptor therapy ${ }^{[29]}$. miRNA-200c can repress the migration and invasion of gastric cancer SGC-7901 cells ${ }^{[30]}$ and enhance 5 -fluorouracil resistance by regulating the expression of dual specificity phosphatase- $6^{[31]}$. And it is reported that miRNAs, including miR-23b, miR-551, miR-1464, and miR-1803, control CTSL gene expression at a post-transcriptional level ${ }^{[32]}$. Nevertheless, the potential role of miRNA-200c in CTSL mediation and drug resistance in A549 cells has yet to be described, and the underlying mechanism has yet to be determined.

On the basis of the previous studies, we aimed to verify the relationship of CTSL, miRNA-200c, and drug resistance and to discuss the potential regulatory mechanism. In the present study, we demonstrated that miRNA-200c can regulate the sensitivity of cells to paclitaxel and EMT. CTSL and miRNA200c are also reciprocally linked in a feedback loop and they reverse paclitaxel resistance by inhibiting EMT in A549/TAX cells.

\section{Materials and methods Materials}

Cell culture reagents and Lipofectamine reagent were purchased from Invitrogen Life Technologies (Carlsbad, CA, USA). Phalloidin was obtained from Sigma-Aldrich (St Louis, MO, USA). The antibodies used in this study were anti-Ncadherin, anti-E-cadherin, anti-Vimentin, and anti-Snail (Santa Cruz Biotechnology, Inc, Santa Cruz, CA, USA); anti-CTSL (Abcam); and anti- $\beta$-actin (MultiSciences Biotech, Hangzhou, China).

\section{Cell lines and culture}

Human lung cancer A549 cell line was purchased from the Type Culture Collection of the Chinese Academy of Sciences, Shanghai, China. A549/TAX cells were purchased from Shanghai MEIXUAN Biological Science and Technology Co, Ltd. A549 cells were cultured in DMEM supplemented with $10 \%$ fetal bovine serum (FBS) and penicillin $(100 \mathrm{U} / \mathrm{mL}) /$ streptomycin $(100 \mathrm{U} / \mathrm{mL})$. A549/TAX cells were cultured in DMEM supplemented with 10\% fetal bovine serum (FBS) and penicillin $(100 \mathrm{U} / \mathrm{mL}) /$ streptomycin $(100 \mathrm{U} / \mathrm{mL})$ and 200 $\mathrm{ng} / \mathrm{mL}$ paclitaxel at $37^{\circ} \mathrm{C}$ in a humidified atmosphere with $5 \%$
$\mathrm{CO}_{2}$.

\section{Quantitative qPCR analyses of miRNA-200c amplifications}

qPCR was performed in an ABI7500 thermocycler with 7500 software v2.03 (Life Technologies Corporation). miRNA quantification: Bulge-loop ${ }^{\mathrm{TM}}$ miRNA qRT-PCR Primer Sets (one RT primer and a pair of qPCR primers for each set) specific for miRNA is designed by RiboBio (Guangzhou, China).

\section{siRNA transfection}

miRNA-200c mimic, miRNA-200c inhibitor, and negative control were obtained from RiboBio (Guangzhou, China). siRNA was mixed with Lipofectamine ${ }^{\circledR} 3000$ Reagent (Invitrogen) and transfected into A549 and A549/TAX cells. After 6h, the supernatant was replaced with a fresh medium containing $10 \% \mathrm{FBS}$ and cultured for another $24 \mathrm{~h}$. siRNA sequences were then used for transfection as follows. miRNA-200c mature chain sequence: 5'-UAAUACUGCCGGGUAAUGAUGGA-3'.

\section{Wound-healing assay}

Cells were grown in six-well plates. After achieving confluency, the cells were scratched with a vpipette tip, rinsed to remove debris, and further incubated with fresh FBS-free culture medium for $24 \mathrm{~h}$. Cell migration images were captured at 0 and $24 \mathrm{~h}$. Wound-healing index, which was determined as percentage, was quantitatively analyzed by using 20 randomly selected distances across the wound at 0 and $24 \mathrm{~h}$ and divided by the distance measured at $0 \mathrm{~h}$.

\section{Transwell invasion assay}

Invasion assay was performed using 24-well Matrigel invasion chambers (BD Biosciences). The cells were trypsinized and reseeded in the upper chamber at a concentration of $1 \times 10^{5} / \mathrm{mL}$ in $200 \mu \mathrm{L}$ of FBS-free DMEM. The lower chamber was composed of $800 \mu \mathrm{L}$ of DMEM and supplemented with $10 \%$ FBS. After $24 \mathrm{~h}$, the cells on the upper surface of the filters were removed, while the cells on the lower surface were fixed with methanol and stained with crystal violet.

\section{Actin cytoskeleton staining}

Cells were grown on coverslips, fixed with $4 \%$ fresh paraformaldehyde for $10 \mathrm{~min}$ at room temperature, permeabilized with $0.1 \%$ Triton X-100 in PBS for $20 \mathrm{~min}$, and blocked with $5 \%$ bovine serum albumin (BSA) at room temperature for $1 \mathrm{~h}$. These cells were subsequently stained with fluorescein isothiocyanate (FITC)-phalloidin for $2 \mathrm{~h}$ at room temperature in the dark, washed, and counterstained with DAPI for $10 \mathrm{~min}$. Confocal microscopy (Carl Zeiss, LSM 710) was employed to observe F-actin distribution.

\section{Cytotoxicity assay}

Methyl thiazolyl tetrazolium (MTT) was used to determine cell viability and proliferation. A549 cells and A549/PTX cells were transfected with miRNA and seeded into 96-well plates at a density of $10^{4}$ cells per well. The cells were cultured for $24 \mathrm{~h}$ in $100 \mu \mathrm{L}$ of complete DMEM. After the cells were pre- 
treated with different paclitaxel concentrations for $24 \mathrm{~h}, 10 \mu \mathrm{L}$ of MTT solution $(5 \mathrm{mg} / \mathrm{mL})$ was added to each well and incubated for $4 \mathrm{~h}$ at $37^{\circ} \mathrm{C}$, and $100 \mu \mathrm{L}$ of $1 \%$ acid was added to each well to dissolve blue formazan crystals. Optical density was measured at $570 \mathrm{~nm}$. These assays were performed in triplicate.

\section{Western blot analysis}

Cells were harvested using a plastic scraper, washed twice with cold PBS, and homogenized in lysis buffer. Protein lysates were quantified using a BCA protein assay kit (Thermo Scientific), loaded, and separated on $10 \%$ or $8 \%$ SDS-PAGE gels. The proteins were transferred onto nitrocellulose blotting membranes. These membranes were blocked with $5 \%$ milkTBST for $1 \mathrm{~h}$, incubated with primary antibodies overnight, and washed thrice. Afterward, the blots were incubated with secondary antibodies for $1 \mathrm{~h}$. Immunoblots were detected in Odyssey Infrared Imaging System (Li-COR Biosciences, Lincoln, NE, USA).

\section{Lentivirus transduction}

A549 cells were seeded in six-well plates in DMEM supplemented with $10 \%$ FBS. The cells were treated with $2 \times 10^{7}$ titration units of lentivirus packaging $24 \mathrm{~h}$ after seeding and synthesized by GeneChem (Shanghai, China). Polybrene $(5 \mathrm{ng} / \mu \mathrm{L})$ was added to enhance the efficiency of viral infections. The cells were harvested $4 \mathrm{~d}$ after infection for either RNA extraction or protein lysate preparation.

\section{Immunofluorescence staining}

The cells were treated $1 \mathrm{~d}$ after they were seeded on coverslips as required for the experiment, fixed with methanol for 10 $\min$ at $4{ }^{\circ} \mathrm{C}$, and permeabilized for $10 \mathrm{~min}$ with $0.1 \%$ Triton $\mathrm{X}-100$. Afterward, the cells were incubated for $1 \mathrm{~h}$ in blocking buffer (1\% BSA and $0.1 \%$ Triton X-100) at $4{ }^{\circ} \mathrm{C}$. For immunofluorescence, the cells were incubated with antibodies against E-cadherin, $\mathrm{N}$-cadherin, and vimentin at $4^{\circ} \mathrm{C}$ overnight. Subsequently, the cells were rinsed thrice with PBS and incubated with the appropriate biotinylated secondary antibodies for 1 h. Alexa Fluor 488 (Molecular Probes, 1:500) and Alexa Fluor 594 goat anti-mouse (Molecular Probes, 1:500) antibodies were used as tertiary antibodies for $1 \mathrm{~h}$. Then, the cells were counterstained with $0.5 \mathrm{ng} / \mathrm{mL}$ DAPI for $15 \mathrm{~min}$ at room temperature, and coverslips were mounted on slides with a VECTASHIELD mounting medium for fluorescence and analysis through confocal microscopy.

\section{Hoechst 33258 staining}

The treated cells were analyzed using a Hoechst staining kit (Sigma). Staining was performed in accordance with the manufacturer's protocol. The cells were washed with PBS, treated with a fixing solution for $10 \mathrm{~min}$, and stained with Hoechst 33258 fluorescent dye for $10 \mathrm{~min}$ at room temperature. Morphological nuclear changes were observed and captured using an inverted fluorescence microscope.

\section{Flow cytometry analysis}

The percentage of apoptotic cells was measured using an Annexin V-(FITC)/PI apoptosis detection kit (KeyGen, Nanjing, China). A total of 10000 cells per sample were examined with a FACSCalibur flow cytometer.

\section{Statistical analysis}

Data were expressed as mean $\pm \mathrm{SD}$, and at least three independent experiments were performed. Differences in the measured variables between experimental and control groups were assessed using Student's $t$-test. $P<0.05$ was considered statistically significant. All analyses were performed using GraphPad Prism 5.0.

\section{Results \\ Feedback loop between CTSL and miRNA-200c}

The miRNA-200c expression was detected through qPCR. The average expression of miRNA-200c was significantly lower in A549/TAX cells, which are paclitaxel-resistant A549 cells, than in A549 cells (Figure 1A). This observation demonstrated that the miRNA-200c expression was downregulated in A549/TAX cells. We then examined the expression of CTSL in these two lung cancer lines (Figure 1B) and found that the CTSL protein levels were higher in A549/TAX cells than in A549 cells. This finding indicated that CTSL might be related to miRNA-200c to some extent.

To further investigate the potential links between CTSL and miRNA-200c, we silenced CTSL in A549/TAX cells and overexpressed CTSL in A549 cells through lentivirus transduction. As shown in Figure 1C and 1D, CTSL knockdown increased miRNA-200c levels in A549/TAX cells, whereas CTSL overexpression decreased miRNA-200c expression in A549 cells. And CTSL overexpression decreased miRNA-200c expression in H1299 cells, which cells with wild-type KRAS (Figure 1F). To further examine the effect of miRNA-200c on CTSL expression, we suppressed miRNA-200c by transfecting A549 cells with an miRNA-200c inhibitor or we overexpressed miRNA200c by transfecting A549/TAX cells with an miRNA-200c mimic. Western blot results suggested that miRNA-200c inhibition evidently increased the CTSL expression in A549 cells, and miRNA-200c mimic decreased the CTSL expression in A549/TAX cells (Figure 1E). Therefore, these results indicated that miRNA-200c and CTSL were reciprocally linked in a feedback loop.

\section{miRNA-200c attenuated paclitaxel resistance in A549 cells}

Considering that miRNA-200c is associated with the development of chemotherapy resistance ${ }^{[2,33]}$, we hypothesized that miRNA-200c may modulate drug resistance in A549 cells. To confirm this hypothesis, we inhibited miRNA-200c in A549 cells or overexpressed miRNA-200c in A549/TAX cells. We then determined the effect of miRNA-200c on $\mathrm{IC}_{50}$ of A549 or A549/TAX cells to paclitaxel through MTT assay, flow cytometry, and Hoechst staining. The MTT assay showed that miRNA-200c knockdown increased $\mathrm{IC}_{50}$ of A549 cells to pacli- 
A
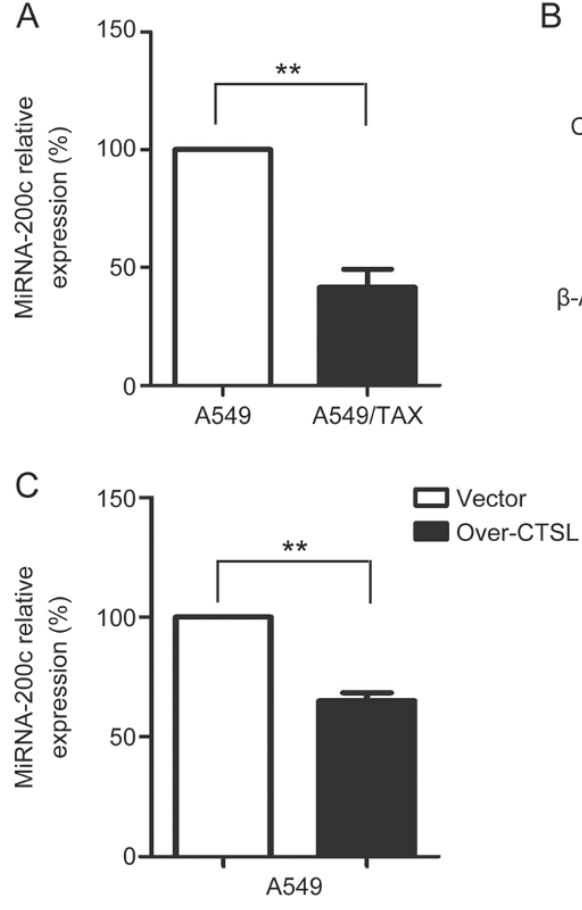

$\mathrm{E}$

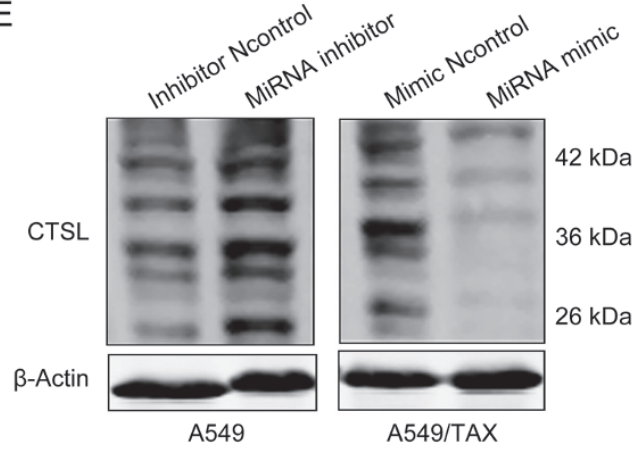

B
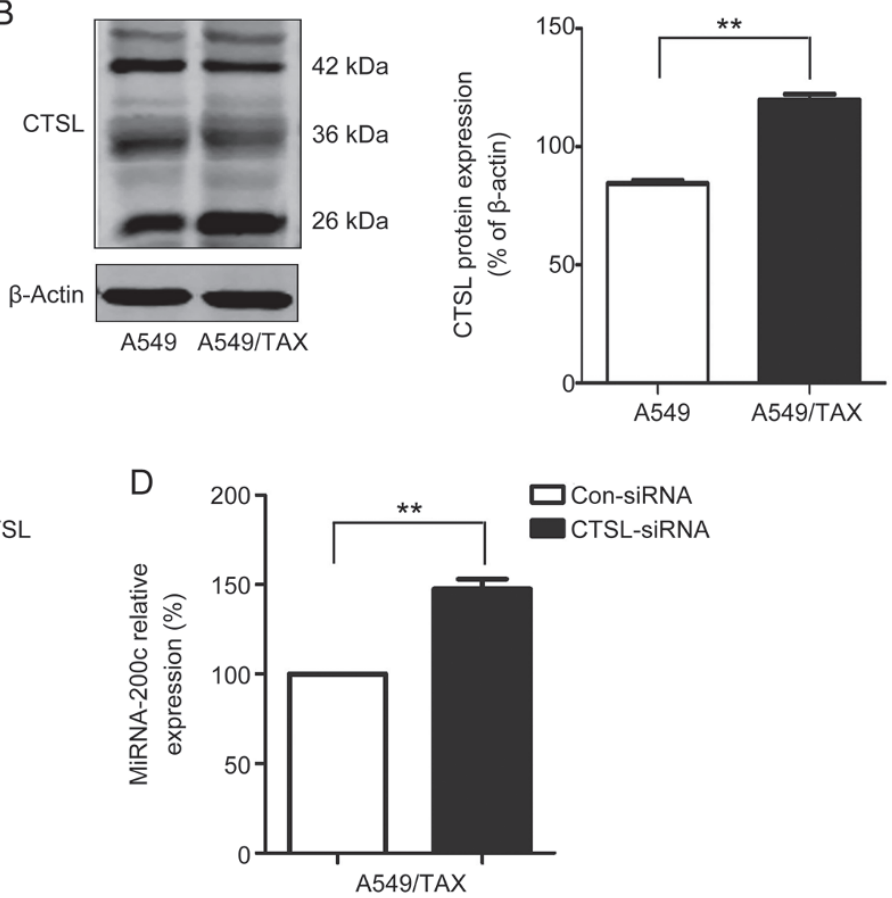
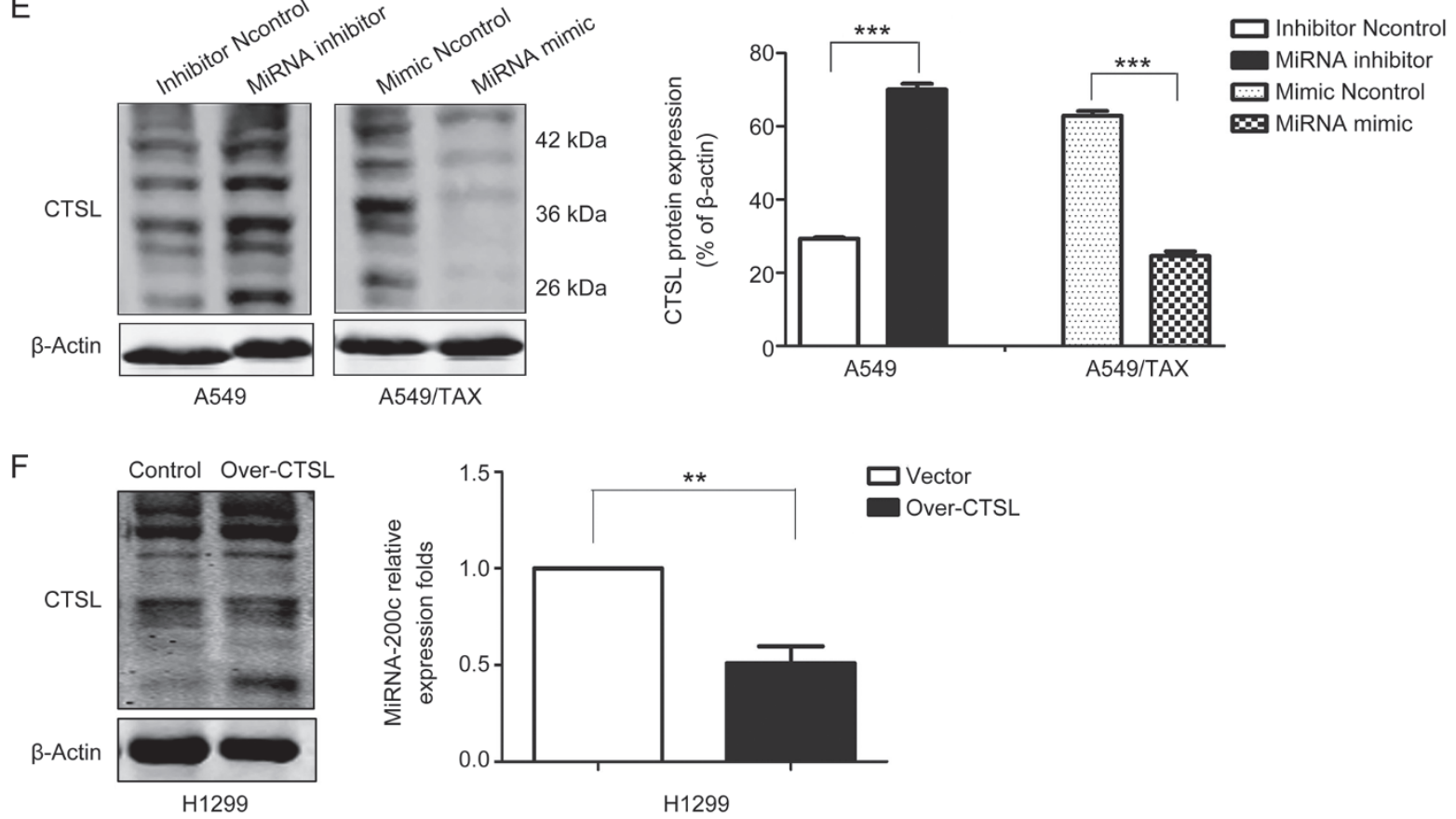

Figure 1. miRNA-200c and CTSL are reciprocally linked in a feedback loop. (A) miRNA-200c levels in A549 cells and A549/TAX cells were measured by qPCR. (B) Western blot was performed to detect the expression of CTSL protein in A549 cells and A549/TAX cells. (C and D) qPCR analysis was performed to determine the level of miRNA-200c of A549 cells infected with LV-Over-CTSL, which targets human CTSL sequence or LV-Vector, and A549/TAX cells transfected with CTSL siRNAs, which target human CTSL sequence or the control siRNA. (E) Western blot was performed to detect the expression levels of CTSL protein in A549 cells transfected with miRNA-200c inhibitor, as the negative control, and A549/TAX cells transfected with miRNA-200c mimic, as the negative control. (F) qPCR analysis was performed to determine the level of miRNA-200c of H1299 cells infected with LVOver-CTSL, which targets human CTSL sequence or LV-Vector. At least three independent experiments were performed. $* P<0.05$, $* * P<0.01$, and ${ }^{* * *} P<0.001$ compared with control.

taxel by approximately 25 -fold, and miRNA-200c overexpression decreased $\mathrm{IC}_{50}$ of A549/TAX cells to paclitaxel by about 20-fold (Figure 2A and 2B). Flow cytometry and Hoechst staining results supported our experimental results (Figure 
A

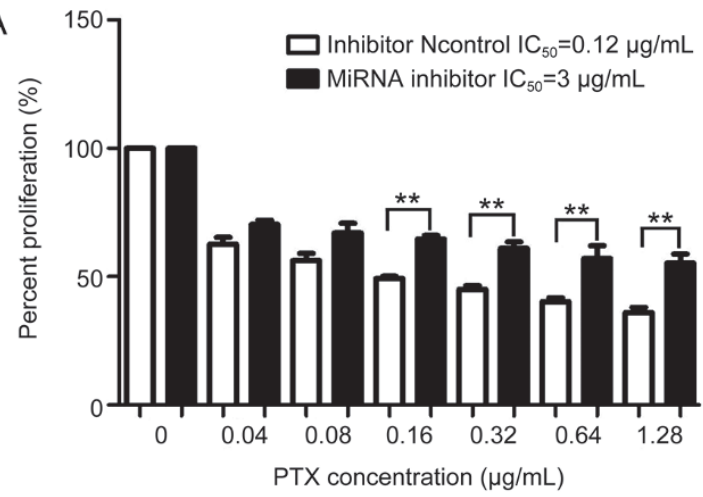

C
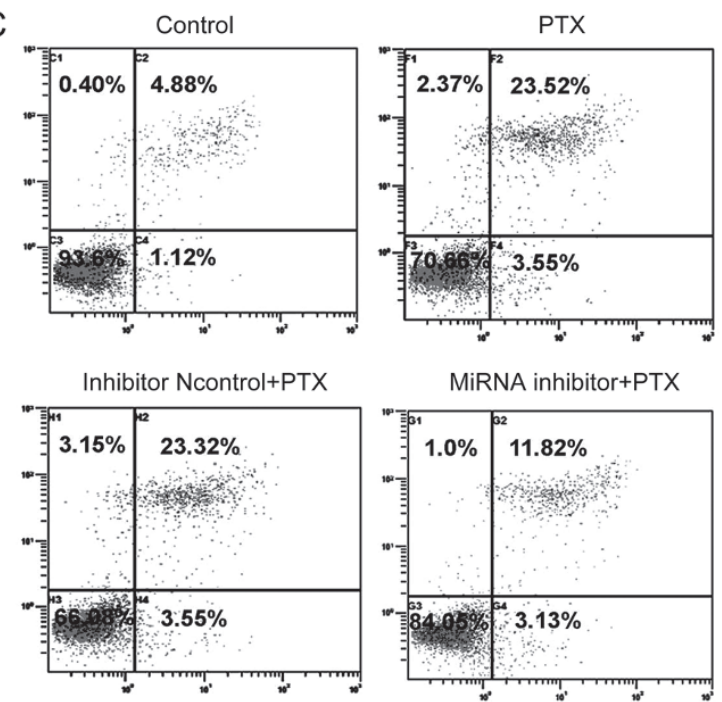

$\mathrm{E}$

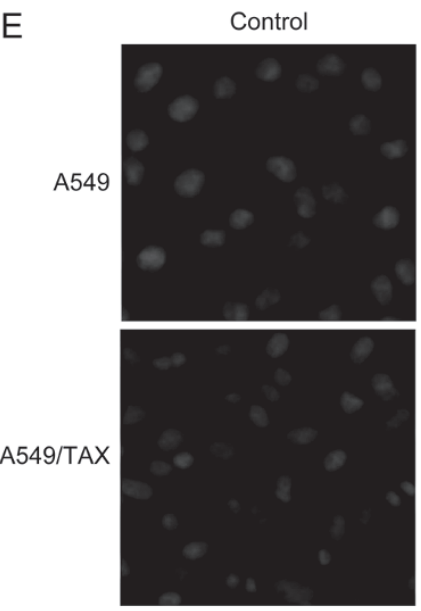

Control
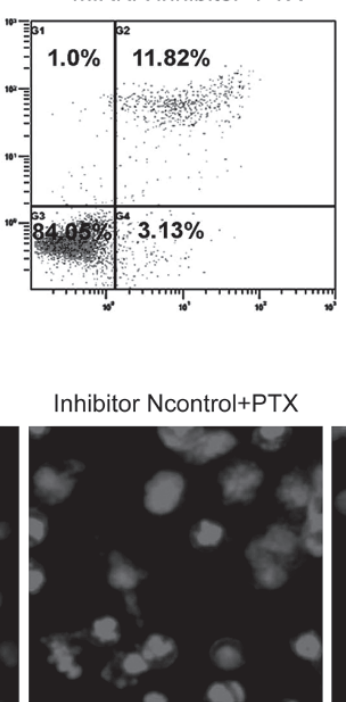

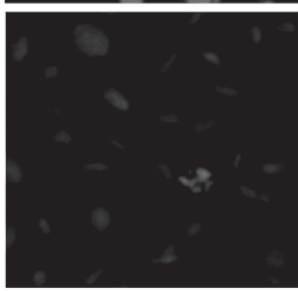

Mimic Ncontrol+PTX

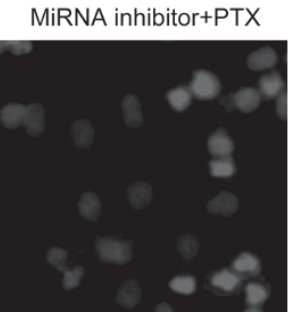

D
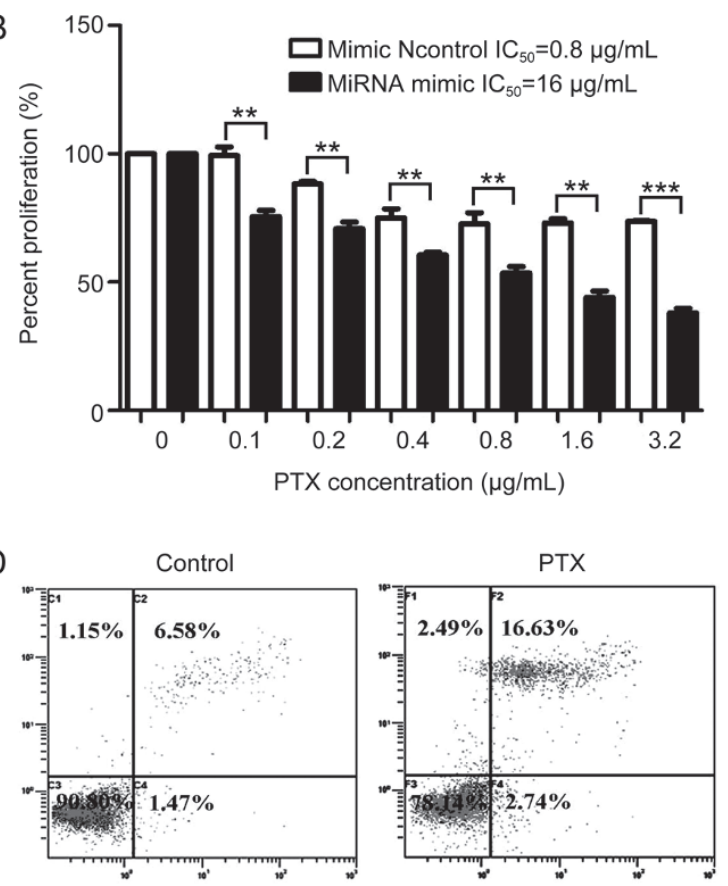

Mimic Ncontrol+PTX

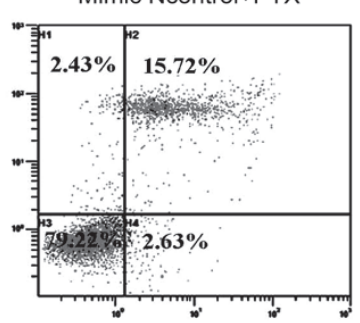

MiRNA mimic+PTX

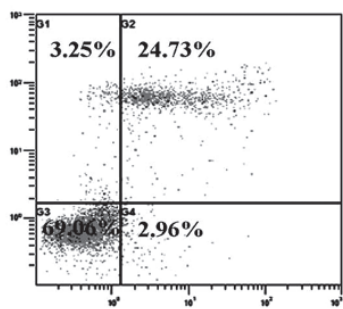

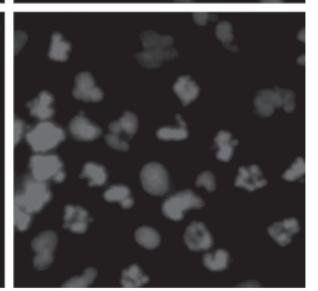

MiRNA mimic+PTX
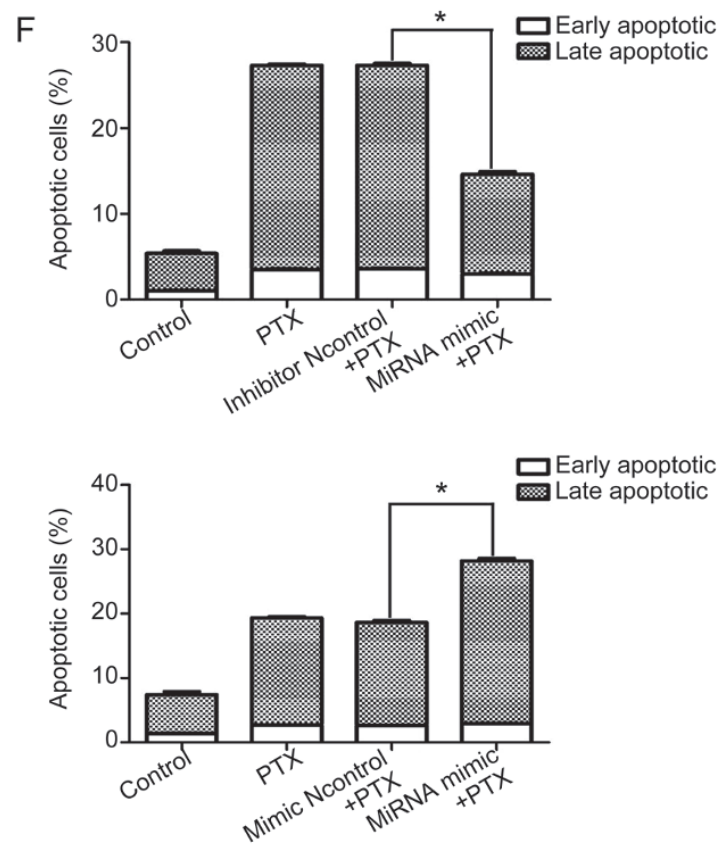

Figure 2. miRNA-200c attenuated paclitaxel resistance in A549 cells. (A and B) MTT assay was performed to determine the $I_{50}$ of $A 549$ cells transfected with miRNA-200c inhibitor, as the negative control, and A549/TAX cells transfected with miRNA-200c mimic, as the negative control. (C-F) The effects of miRNA-200c on sensitivity of A549 or A549/TAX cells to paclitaxel by flow cytometry and Hoechst staining. At least three independent experiments were performed. ${ }^{*} P<0.05,{ }^{* *} P<0.01$, and ${ }^{* * *} P<0.001$ compared with control group. 
2C-2E). And these results confirmed that miRNA-200c plays a vital role in the regulation of paclitaxel resistance in A549 cells.

miRNA-200c was involved in the progression of CTSL-mediated drug resistance in $\mathrm{A} 549$ cells

Our previous work demonstrated that CTSL can regulate drug resistance by mediating EMT through its effects on the expression of EMT-associated transcription factors, such as Snail, Slug, ZEB1, and ZEB2 ${ }^{[12]}$. Therefore, we hypothesized that whether miRNA-200c can reverse paclitaxel resistance which induced by CTSL. To confirm this hypothesis, we silenced CTSL in A549/TAX cells and overexpressed CTSL in A549 cells through lentivirus transduction. We then inhibited or overexpressed miRNA-200c in these two cells. Then, we determined the effect of CTSL knockdown and miRNA-200c inhibition on $\mathrm{IC}_{50}$ of A549/TAX cells to paclitaxel through MTT assay and Hoechst staining. Our results revealed that the $\mathrm{IC}_{50}$ of the treated A549/TAX cells to paclitaxel increased by approximately 3.9-fold compared with CTSL-inhibited A549/ TAX cells (Figure 3A). MTT and Hoechst assay results also suggested that the forced expression of CTSL and miRNA-200c decreased $\mathrm{IC}_{50}$ of A549 cells to paclitaxel by approximately 5.8fold compared with CTSL-overexpressed A549 cells (Figure $3 \mathrm{~B}$ and $3 \mathrm{C})$. These findings further confirmed that miRNA200c participates in the progression of CTSL-mediated drug resistance in A549/TAX cells, and miRNA-200c can reverse paclitaxel resistance induced by CTSL.

miRNA-200c reversed paclitaxel resistance by suppressing cell invasion and migration

The acquisition of paclitaxel resistance is associated with

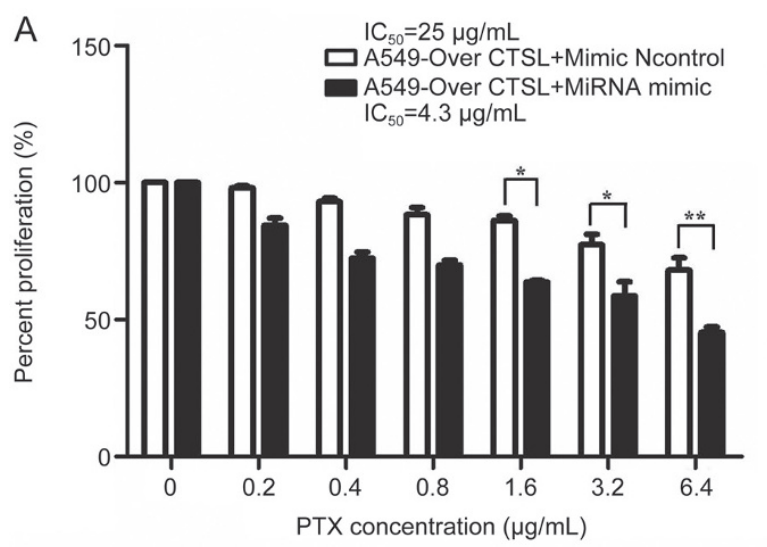

C

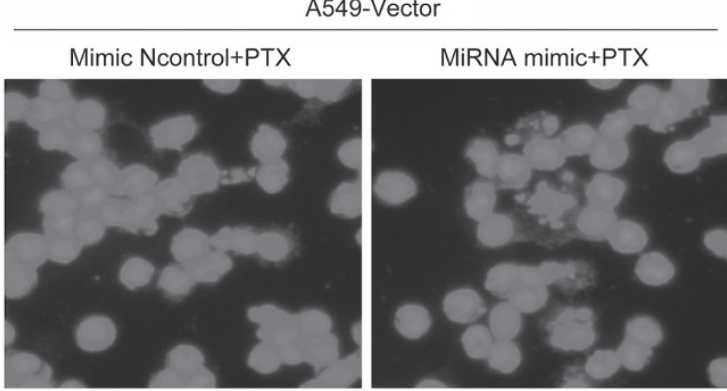

A549/TAX-Vector
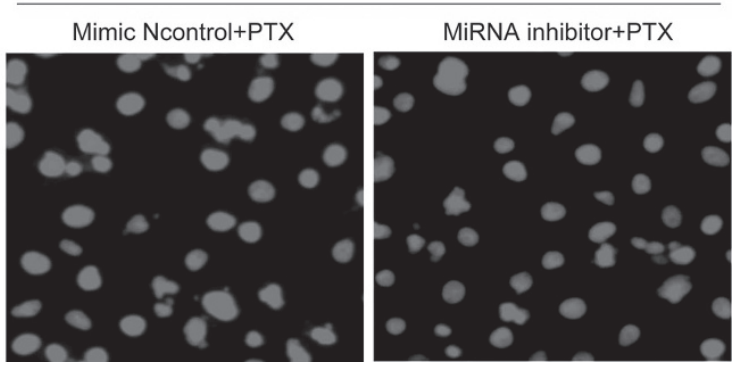

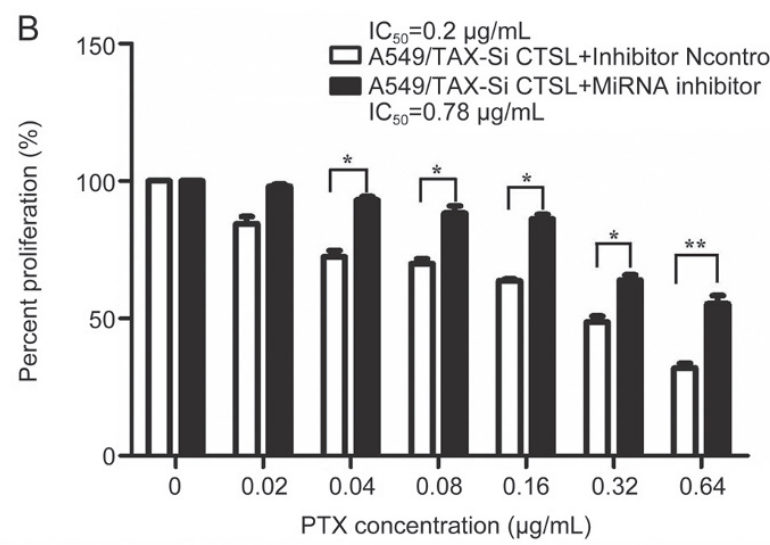

A549-Vector Cathepsin L

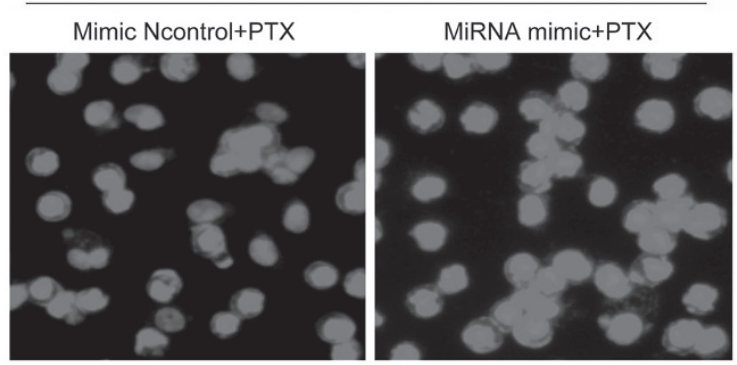

A549/TAX-Si Cathepsin L

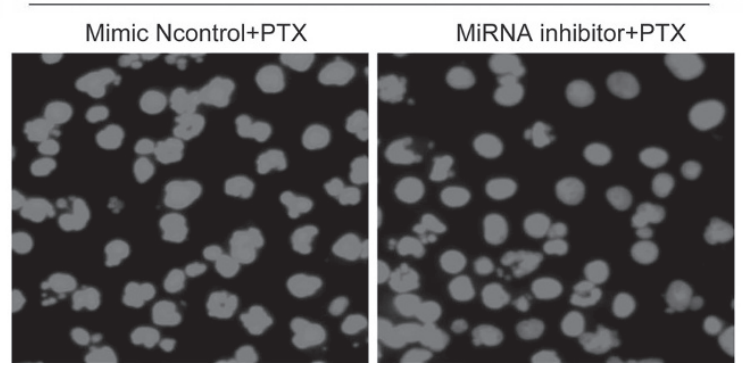

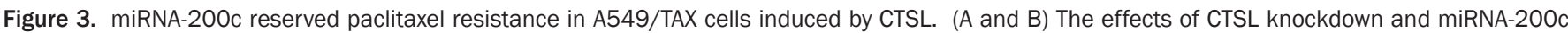

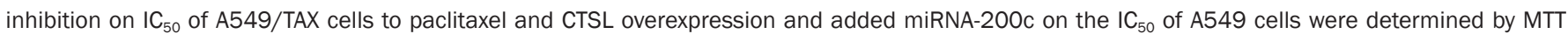

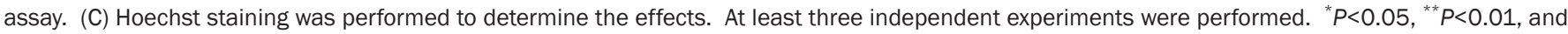
${ }^{* * *} P<0.001$ compared with control group. 
an aggressive and invasive phenotype in prostate cancer ${ }^{[34]}$. Consistent with our previous findings ${ }^{[12]}$, this phenomenon illustrates a possible relationship between drug resistance and invasive potential. Our study hypothesized that miRNA200c can regulate paclitaxel resistance by inhibiting cell invasion and migration. To verify this hypothesis, we conducted wound-healing and Transwell assays to detect the effects of miRNA-200c on cell invasion and migration. The results showed that miRNA-200c knockdown enhanced the invasion and migration of A549 cells, and miRNA-200c overexpression decreased the invasion and migration of A549/TAX cells (Figure $4 \mathrm{~A}$ and $4 \mathrm{~B}$ ). Furthermore, the suppression of miRNA200c noticeably induced an increase in lamellipodia and stress fibers in A549 cells. The overexpression of miRNA-200c evidently suppressed actin remodeling associated with A549/ TAX cells motility (Figure 4C). Therefore, miRNA-200c regulates paclitaxel resistance by suppressing cell invasion and migration.

\section{CTSL cooperated with miRNA-200c to mediate cell invasion and migration}

To investigate the potential roles of CTSL and miRNA-200c in mediating cell invasion and migration, we overexpressed CTSL in A549 cells and inhibited CTSL in A549/TAX cells, then we subjected them to wound healing and Transwell assays. We observed that the overexpression of CTSL could induce the invasion and migration of A549 cells, and the suppression of CTSL could inhibit the invasion and migration of A549/TAX cells. Then, we transfected the miRNA200c mimic or inhibitor into these two cells and examined the cells through wound healing and transwell assays to evaluate cell migration and invasion. In Figure 5A and 5B, miRNA200 c overexpression decreased the invasion and migration in CTSL-overexpressed A549 cells. By contrast, miRNA-200c suppression increased the invasion and migration in CTSLinhibited A549/TAX cells. These results indicated that CTSL and miRNA-200c could regulate paclitaxel resistance in A549 cells by controlling cell invasion and migration.

\section{miRNA-200c played a vital role in mediating EMT in A549 cells}

EMT is a key element in cell migration, invasion, and drug resistance in several cancer types ${ }^{[35]}$. Blocking and reversing EMT may be important strategies to overcome chemotherapy resistance. On the basis of the experimental results, we hypothesized that miRNA-200c could regulate drug resistance by mediating EMT. Western blot was performed to determine the effect of miRNA-200c on the expression of EMT marker proteins. Our results demonstrated that miRNA-200c knockdown decreased the expression of epithelial markers, namely, E-cadherin and cytokeratin 18, and increased the expression of mesenchymal markers, namely, $\mathrm{N}$-cadherin and vimentin. And miRNA-200c overexpression upregulated the level of epithelial markers and downregulated the level of mesenchymal markers (Figure 6B and 6C). Furthermore, miRNA-200c knockdown promoted the expression of Snail in A549 cells, whereas miRNA-200c overexpression reduced the expression of Snail in A549/TAX cells (Figure 6B). Our immunofluorescence results supported our Western blot results (Figure 6A) and these results indicated that miRNA-200c regulates paclitaxel resistance by inhibiting EMT.

\section{miRNA-200c suppressed EMT induced by CTSL}

CTSL can regulate cisplatin and paclitaxel resistance by inhibiting EMT, and our results also suggested that miRNA200c could regulate paclitaxel resistance by preventing EMT. These observations confirmed that miRNA-200c may reverse EMT induced by CTSL. To confirm the role of miRNA-200c in mediating EMT, which was induced by CTSL, we silenced CTSL in A549/TAX cells and overexpressed CTSL in A549 cells through lentivirus transduction, then we inhibit miRNA200c or overexpressed miRNA-200c in these two cells. Western blot results showed that CTSL knockdown in A549/ TAX cells reduced the expression of N-cadherin, vimentin, and Snail and increased the expression of E-cadherin. However, the suppression of miRNA-200c noticeably induced an increase in the expression of N-cadherin, vimentin, and Snail and a decrease in the expression of E-cadherin. Furthermore, the CTSL overexpression in A549 cells promoted the expression of N-cadherin, vimentin, and Snail and decreased the expression of E-cadherin. The miRNA-200c overexpression could reverse these changes, that is, its overexpression decreased the expression of $\mathrm{N}$-cadherin, vimentin, and Snail and increased the expression of E-cadherin (Figure 7A and 7B). Our immunofluorescence results confirmed our experimental observations (Figure 7B). Hence, these results suggested that CTSL and miRNA-200c regulate EMT in lung cancer cells by controlling EMT-associated transcription factors. Therefore, we draw a conclusion that miRNA-200c could decrease the expression of CTSL, and there are reciprocally linked in a feedback loop between CTSL and miRNA-200c, they reverse paclitaxel resistance by inhibiting epithelial-mesenchymal transition in A549/TAX cells (Figure 7D).

\section{Discussion}

The question addressed by the present study was whether an miRNA-200c link exists between CTSL expression and drug resistance. To address this question, we quantified CTSL and miRNA-200c levels in lung cancer cells and found that CTSL expression was inversely correlated with miRNA-200c expression. The forced CTSL expression in low-CTSL-expressing A549 cells inhibited the miRNA-200c expression, and the interference with CTSL expression in high-CTSL-expressing A549/TAX cells promoted the miRNA-200c expression. The miRNA-200c inhibitor evidently increased the CTSL expression in A549 cells, and the miRNA-200c mimic decreased the CTSL expression in A549/TAX cells. Therefore, we draw a conclusion that there are reciprocally linked in a feedback loop between CTSL and miRNA-200c. In addition, A549 cells harbors KRAS mutations, whereas miRNA-200c has been reported to target the KRAS gene ${ }^{[36]}$. Taken together, we selected another human lung cancer cell lines, H1299 cells with wild type KRAS, qRT-PCR results suggested that CTSL 
A

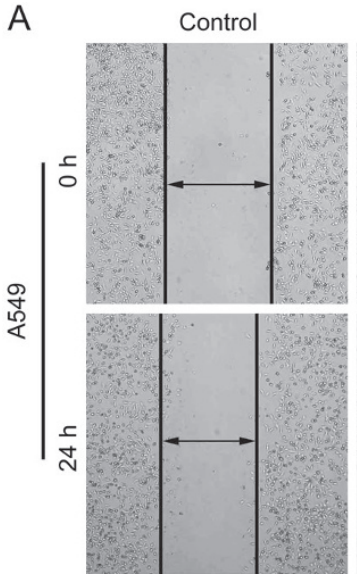

B

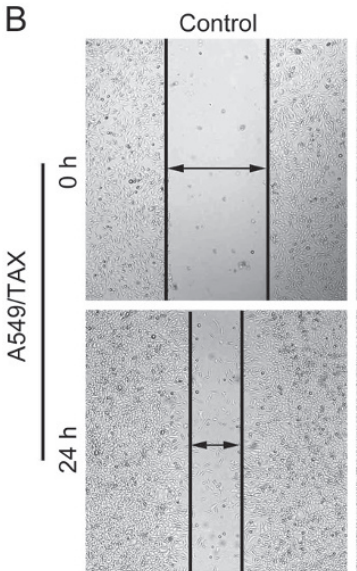

Inhibitor Ncontrol

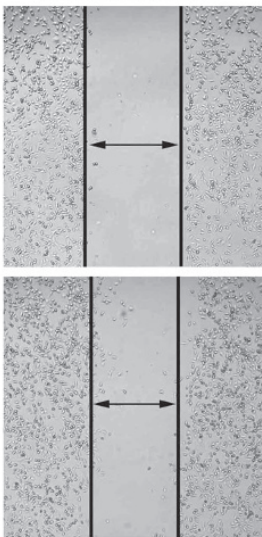

Inhibitor Ncontrol

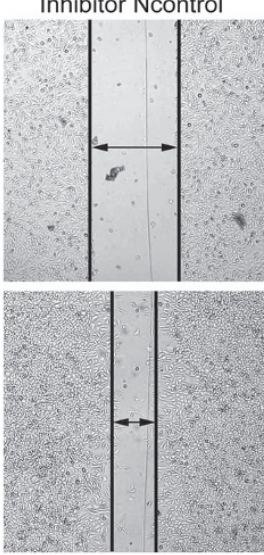

MiRNA inhibitor

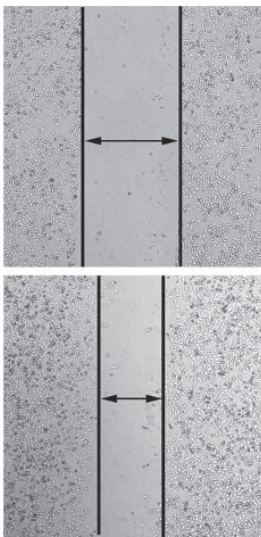

C
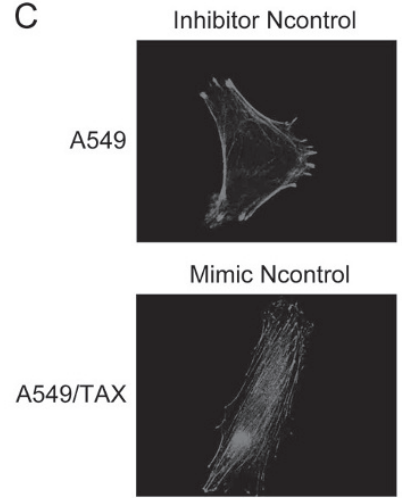

Mimic Ncontrol

MiRNA inhibitor

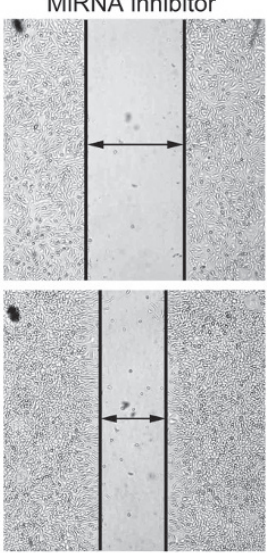

A549

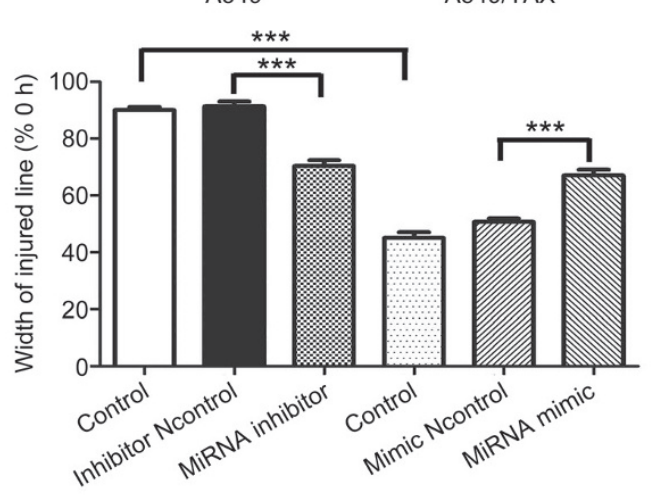

D

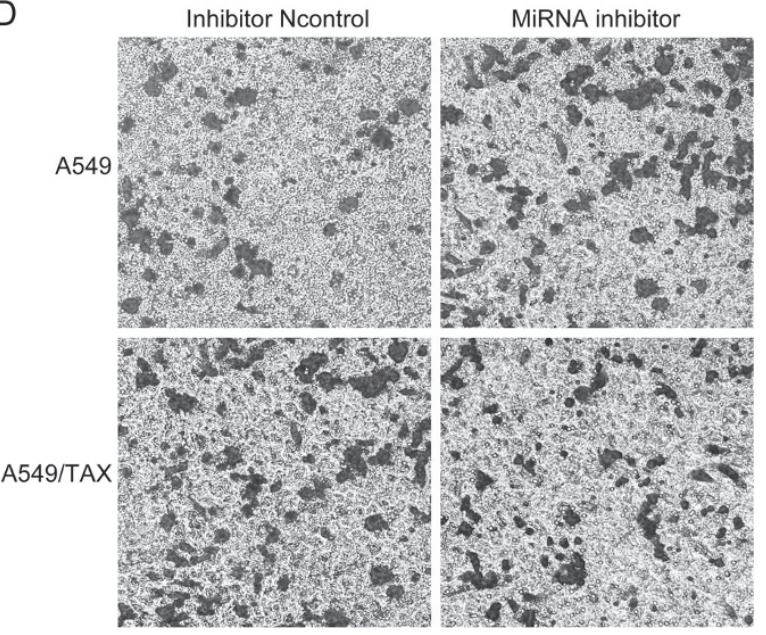

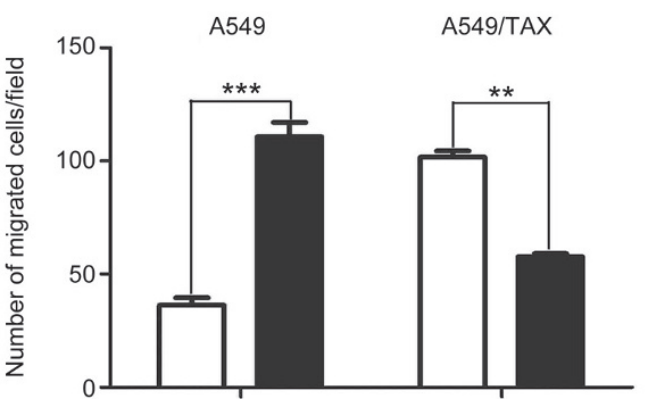

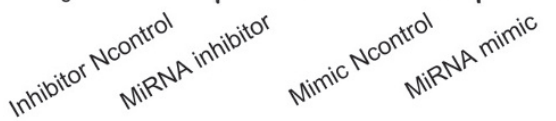

Figure 4. miRNA-200c suppressed cell invasion and migration in A549 and A549/TAX cells. (A and B) The ability of cell migration was evaluated by wound-healing assay. Images were captured using a $4 \times$ objective lens. (C) The ability of cell invasion was evaluated by transwell assay. Images were captured using a 10x objective lens. (D) Representative phalloidin staining for actin-cytoskeleton in A549 transfected with miRNA-200c inhibitor or negative control and A549/TAX cells transfected with miRNA-200c mimic or negative control. Spreading of cells was analyzed after plating on collagencoated slides. Cells were fixed, stained with phalloidin, and visualized at indicated time points by fluorescent microscopy. Images were captured using a $63 \times$ objective lens. At least three independent experiments were performed. ${ }^{*} P<0.05,{ }^{* *} P<0.01$, and ${ }^{* * *} P<0.001$ compared with control group.

overexpression decreased miRNA-200c expression in H1299 cells (Figure 1F). These results indicated that the relationship of miRNA-200c and CTSL is applicable to multiple types of lung cancer cell lines including cells with wild-type KRAS. CTSL may be a potential target of miRNA-200c to regulate drug resistance. 
A
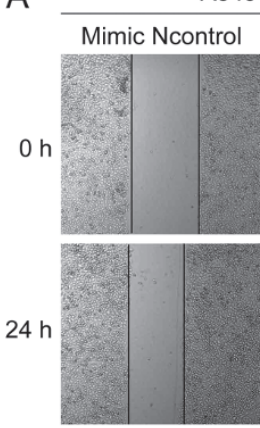

B

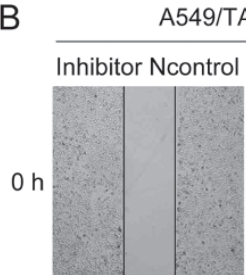

A549/TAX-Vector
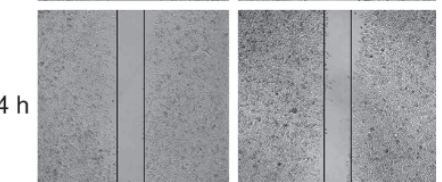

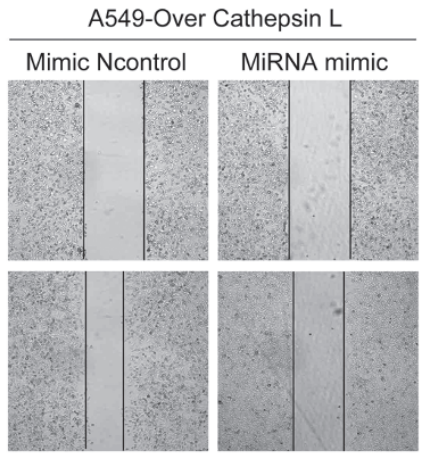

A549/TAX-Si Cathepsin L

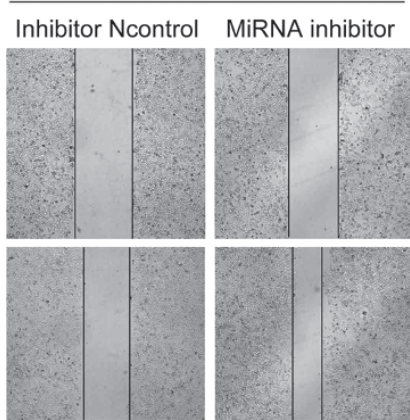

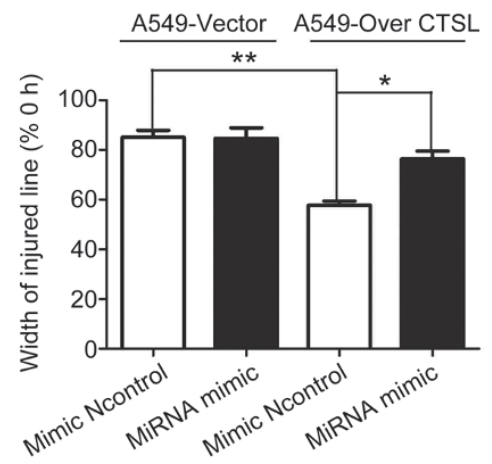

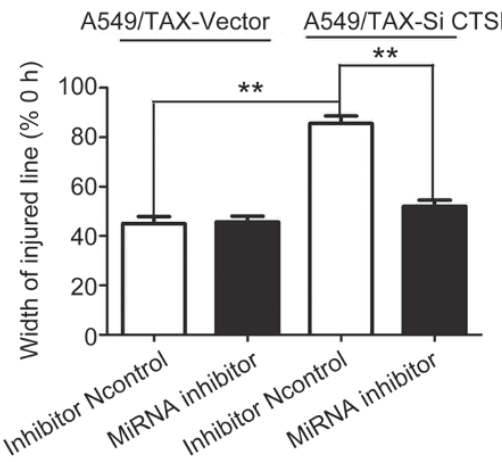

C

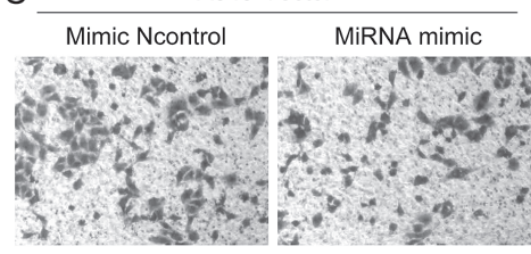

A549/TAX-Vector

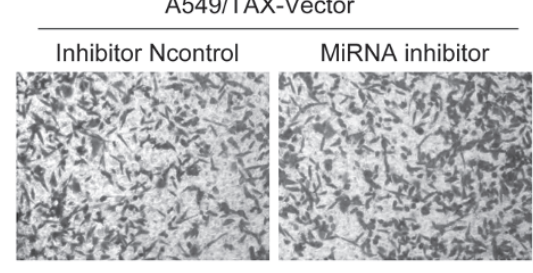

A549-Over Cathepsin L

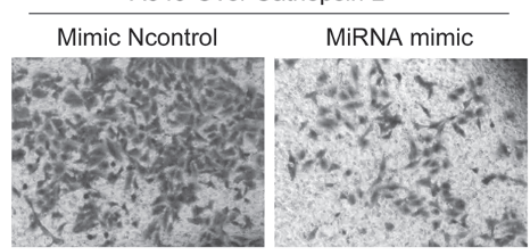

A549/TAX-Si Cathepsin L

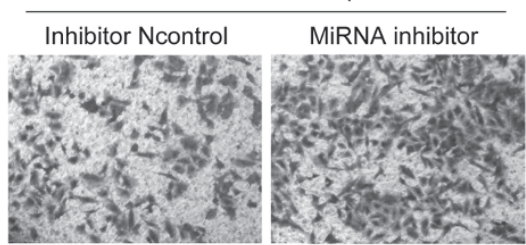

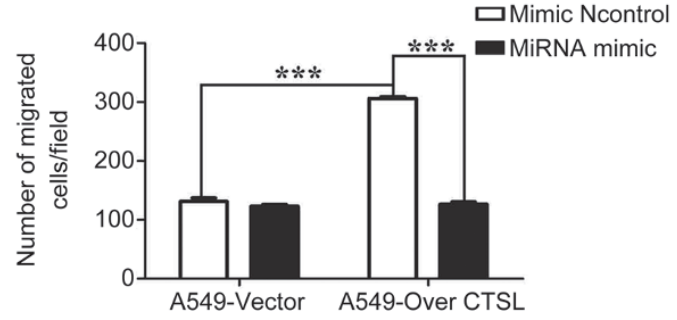

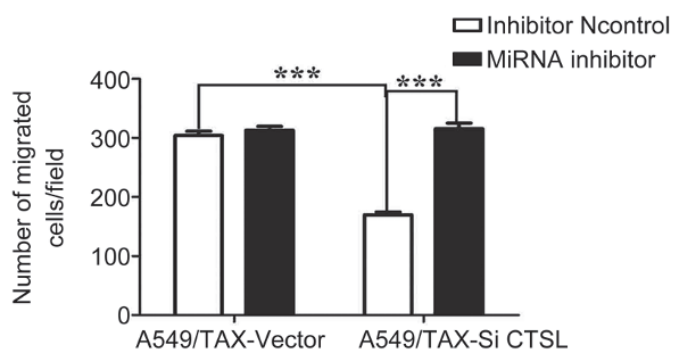

Figure 5. CTSL cooperated with miRNA-200c in mediating cell invasion and migration. (A and B) Effects of CTSL overexpression and miRNA-200c mimic in A549 cells and CTSL inhibition and miRNA-200c inhibitor in A549/TAX cells on cell migration. Images were captured using a 4× objective lens. (C) Effects of CTSL overexpression and miRNA-200c mimic in A549 cells and CTSL inhibition and miRNA-200c inhibitor in A549/TAX cells on cell invasion. Images were captured using a $4 \times 0$ objective lens. At least three independent experiments were performed. ${ }^{*} P<0.05,{ }^{* *} P<0.01$, and ${ }^{* * *} P<0.001$ compared with control group.

Chemotherapy plays an important role in lung cancer treatment, but the existence of drug resistance restricts therapeutic efficacy. Therefore, novel therapeutic targets must be developed to reinforce the efficacy of cancer chemotherapy. CTSL is implicated in malignant transformation ${ }^{[3]]}$ and is upregulated in various drug-resistant cancer cells. Studies have also proposed that CTSL is involved in the regulation of drug resistance. Zheng et al revealed that CTSL inhibition enhances the availability of cytoplasmic and nuclear protein drug targets, including estrogen receptor- $\mathrm{a}, \mathrm{Bcr}-\mathrm{Abl}$, topoisomerase-IIa, histone deacetylase 1 , and androgen receptor ${ }^{[9]}$. CTSL suppression also improves gefitinib resistance in non-small cell lung cancer ${ }^{[14]}$, and CTSL knockdown can increase the sensitivity of ovarian cancer cells to paclitaxe ${ }^{[38]}$. Our previous study also suggested that CTSL may be a novel therapeutic target to prevent tumor cells from becoming resistant to chemotherapy and 
A

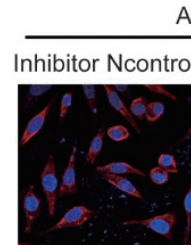

A549
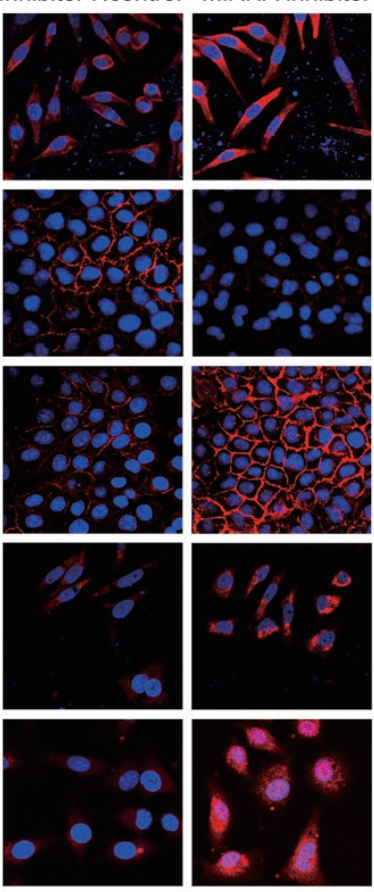

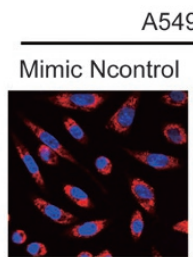

A549/TAX
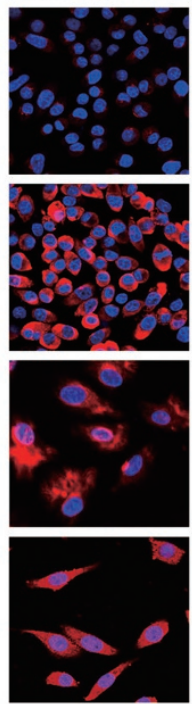

B
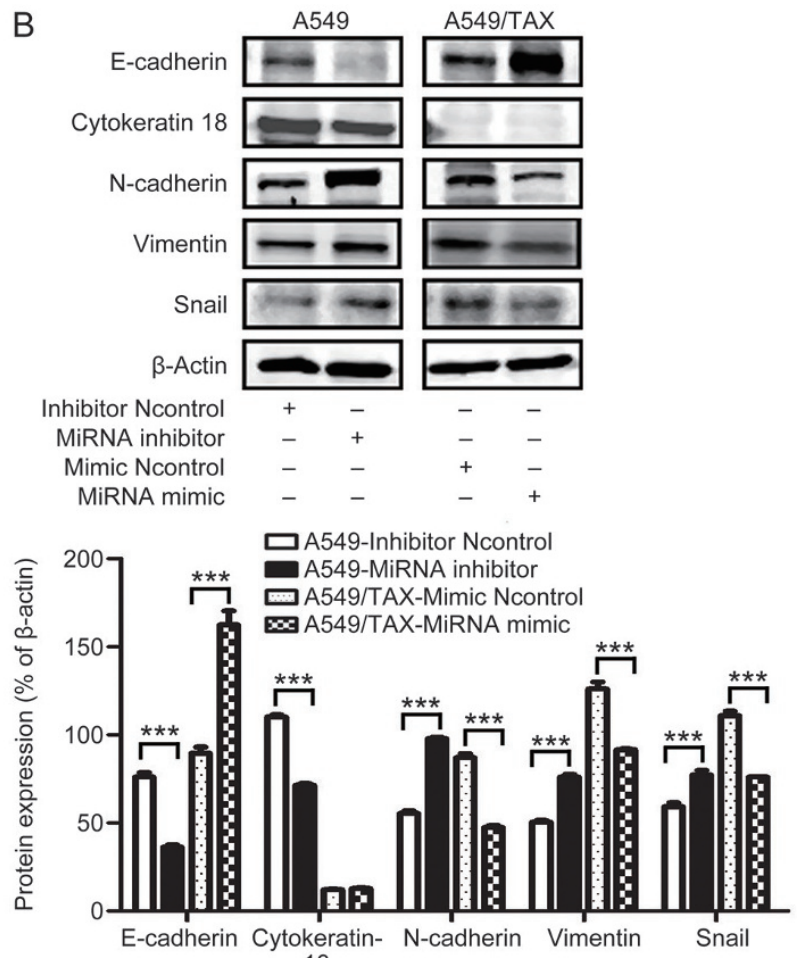

E-cadherin Cytoker 18
C

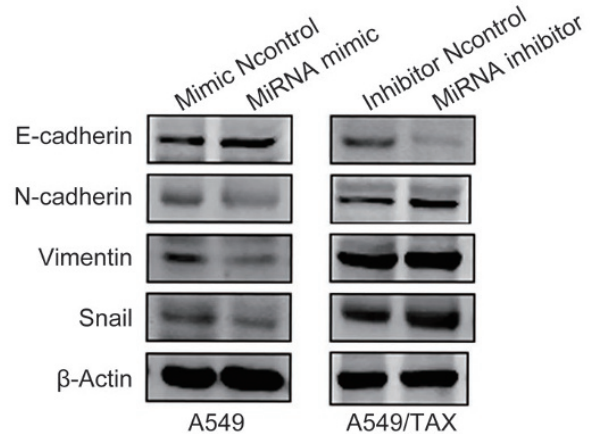

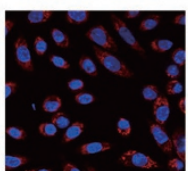
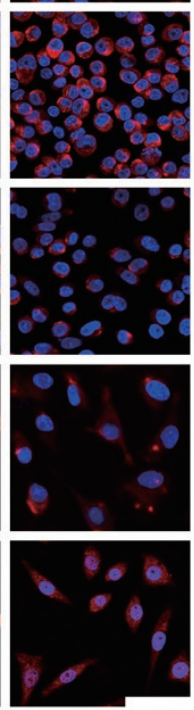

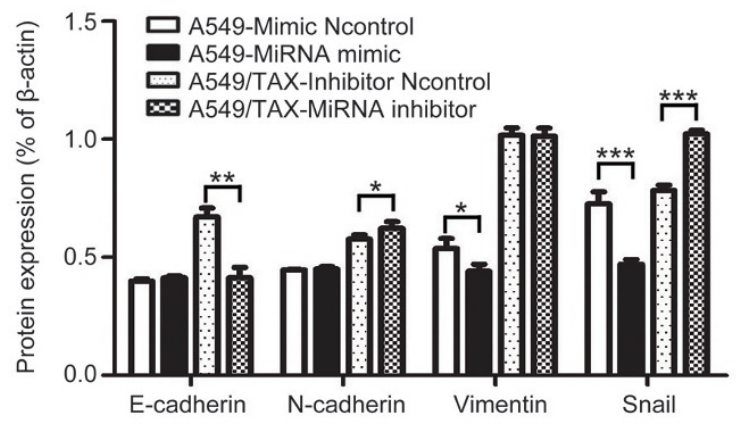

Figure 6. miRNA-200c regulates paclitaxel resistance by suppressing EMT in A549/TAX cells. (A) Effects of miRNA-200c on CTSL, Snail, E-cadherin, $\mathrm{N}$-cadherin, and vimentin were subjected to immunofluorescent microscopy. Images were captured using a $63 \times$ objective lens. (B and C) Effects of miRNA-200c on E-cadherin, N-cadherin, cytokeratin-18, Snail, and vimentin were assessed by Western blot analysis. At least three independent experiments were performed. ${ }^{*} P<0.05,{ }^{* *} P<0.01$, and ${ }^{* * *} P<0.001$ were compared with control.

to reinforce the efficiency of paclitaxel and cisplatin against lung cancer ${ }^{[12]}$. As previously reported, miRNA-200c and other factors in its regulatory pathway are correlated with chemoresistance $^{[39,40]}$. Chang et al reported that miRNA-200c regulates the sensitivity of chemotherapy to cisplatin in gastric cancer by possibly targeting $\mathrm{RhoE}^{[41]}$. Ma et al revealed that miR-200c overexpression can decrease the colony formation, invasion, and chemoresistance of PCSCs ${ }^{[42]}$. However, the exact role of miR-200c in mediating drug resistance in A549 cells, its relationship with CTSL, and its underlying mechanism have yet to be elucidated. In our studies, we confirmed that miRNA200c attenuated the sensitivity of A549/TAX cells to paclitaxel, and miRNA-200c participated in the progression of CTSLmediated drug resistance in A549/TAX cells. miRNA-200c could reverse CTSL-induced paclitaxel resistance.
Several mechanisms, including drug efflux, drug inactivation, drug target alteration, DNA damage repair, and cell death inhibition, promote or enable drug resistance ${ }^{[43]}$. The role of EMT in cancer drug resistance is an emerging research area $^{[44,45]}$. EMT has also been established as a mechanism that provides tumor cells with abilities essential for drug resistance ${ }^{[46,47]}$. EMT presumably occurs through multiple signaling pathways affected by microenvironments. The identification of miRNAs that regulate multiple EMT pathways is also a useful approach that can reveal potential therapeutic targets to help improve clinical outcomes in cancer treatment. The repression of the transcription of miRNA-200 family members by Nanog in colon cancer cells induces $\mathrm{EMT}^{[48]}$, and smad3 can regulate EMT via the miRNA-200 pathway ${ }^{[49]}$. Our early research showed that CTSL downregulation inhibits EMT to 
A

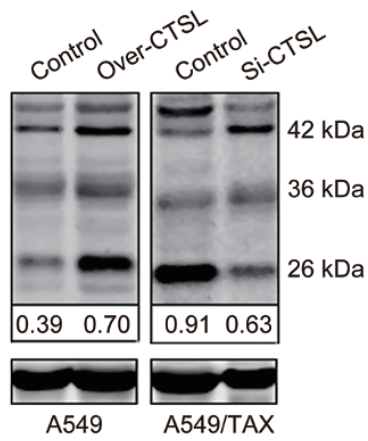

D

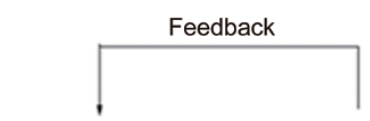

miRNA-200c - Cathepsin L

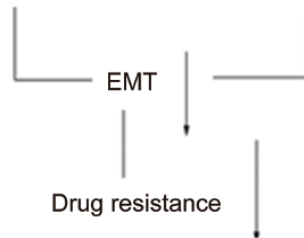

B

$\begin{array}{rllllll}\text { Inhibitor Ncontrol } & - & - & - & - & - & - \\ \text { MiRNA inhibitor } & - & - & - & - & - & - \\ \text { Mimic Ncontrol } & - & + & - & - & + & - \\ \text { MiRNA mimic } & - & - & + & - & - & +\end{array}$

E-cadherin

$\mathrm{N}$-cadherin
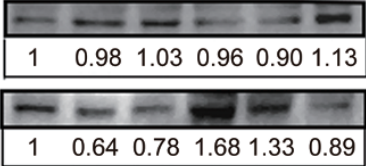

Snail

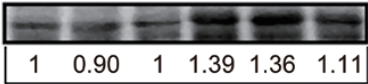

Vimentin

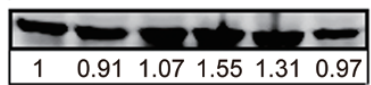

CTSL

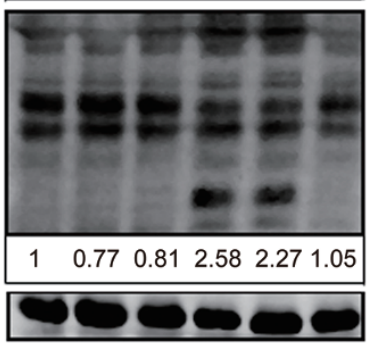

GAPDH
A549/TAX-Vector A549/TAX-Si CTSL
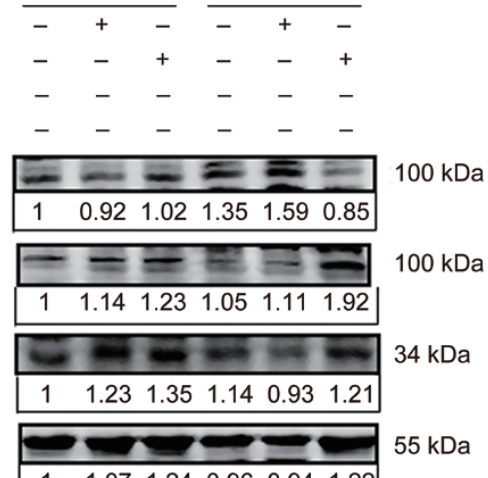

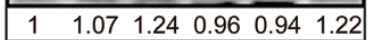
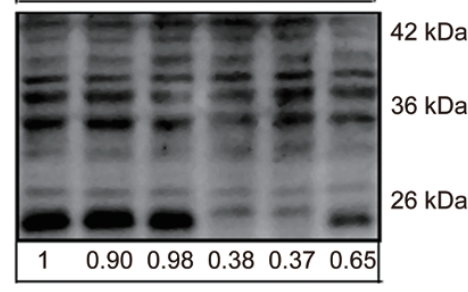

$36 \mathrm{kDa}$

$26 \mathrm{kDa}$

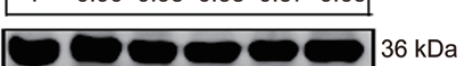

C

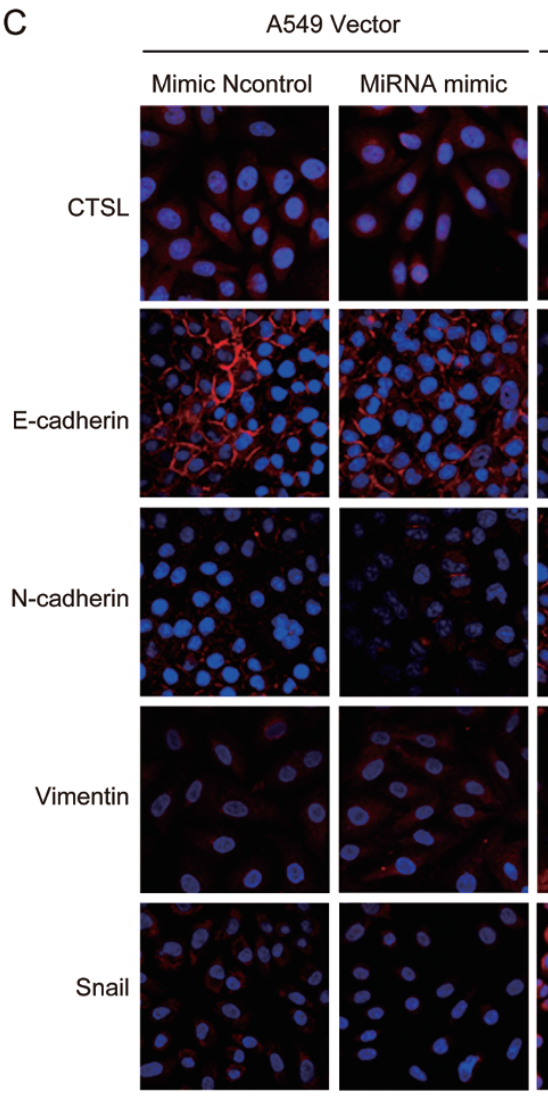

A549 Vector
A549-Over Cathepsin L
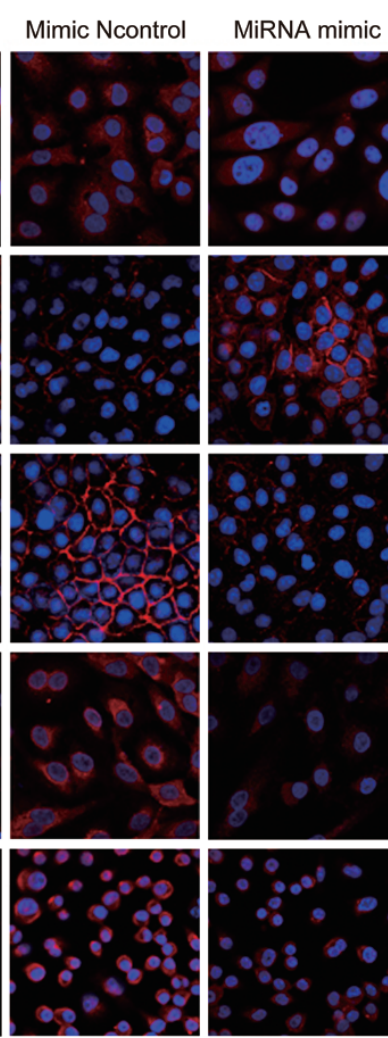

A549 Vector

Inhibitor Ncontrol MiRNA inhibitor Inhibitor Ncontrol MiRNA inhibitor
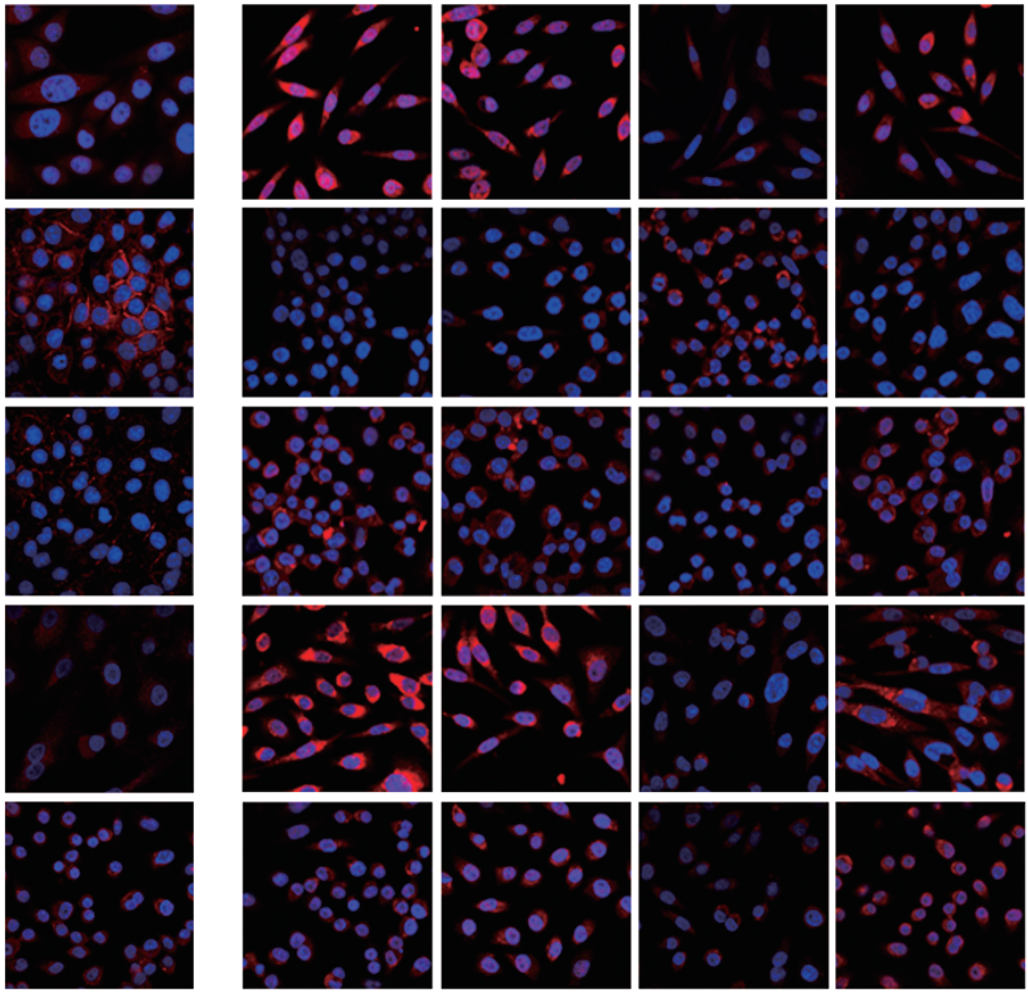

Figure 7. miRNA-200c suppresses CTSL-induced EMT. (A) The effect of CTSL overexpression on A549 cells and the effect of CTSL inhibition on A549/ TAX cells. (B) Effect of CTSL overexpression and miRNA-200c mimic in A549 cells and effect of CTSL inhibition and miRNA-200c inhibitor on E-cadherin, $\mathrm{N}$-cadherin, Snail, and vimentin levels in A549/TAX cells were assessed by Western blot analysis. (C) Effects of CTSL overexpression and miRNA-200c mimic in A549 cells and CTSL inhibition and miRNA-200c inhibitor in A549/TAX cells on CTSL, Snail, E-cadherin, N-cadherin, and vimentin levels were subjected to immunofluorescent microscopy. (D) A schematic figure to demonstrate the regulatory link of miRNA-200c, CTSL and EMT. Images were captured using a $63 \times$ objective lens. At least three independent experiments were performed. 
suppress cancer invasion and migration ${ }^{[11]}$. Thus, we inferred that the effects of miRNA-200c and CTSL may regulate paclitaxel resistance by inhibiting EMT in A549 cells. Our results demonstrated that miRNA-200c inhibition decreased the sensitivity of A549 cells to paclitaxel by suppressing EMT and by increasing the expression of EMT-associated transcription factors. Otherwise, miRNA-200c overexpression increased the sensitivity of A549/TAX cells to paclitaxel and reversed the EMT phenotype. Furthermore, miRNA-200c suppressed paclitaxel resistance by inhibiting EMT in A549/TAX cells, which could be induced by CTSL.

EMT is an essential process in cancer drug resistance and is an emerging area in research. EMT-promoting transcription factors, such as Snail/Slug/Twist and ZEB1/2, directly bind to E-cadherin promoter and repress its transcription. EMT is involved in the activation of transcription factors that interact with epigenetic regulators to control the expression of EMT marker proteins ${ }^{[29]}$. Several transcription factors, such as those of the Snail/Slug family, Twist, and ZEB1/2, function as molecular switches for the EMT program ${ }^{[29]}$. EMT has also been established as a mechanism that provides tumor cells with abilities essential for paclitaxel resistance in many cells. ZEB2 is an important EMT-transcription factor, and in our laboratory, we have confirmed that CTSL is initially identified as a novel EMT regulator, it can regulate drug resistance by mediating EMT through its effects on the expression of EMT-associated transcription factors, Snail, Slug, ZEB1, and ZEB2 $^{[11,12]}$. Also miRNA-200 targets ZEB2, it functions as a key regulator of EMT in numerous cancers and promotes an epithelial phenotype by inhibiting several EMT genes, including ZEB1 and ZEB2 ${ }^{[50,51]}$. miRNA-200c inhibits the resistance of A549/TAX cells to paclitaxel by inhibiting EMT whether by suppressing the expression of ZEB2. And, we just suggested that Snail was significantly involved in the regulation of EMT by CTSL and miRNA-200c, but further research should be performed to confirm that whether other EMT-associated transcription factors were involved in this progression.

In summary, our study confirmed that CTSL and miRNA200c were reciprocally linked in a feedback loop, and miRNA200c could inhibit the paclitaxel resistance of A549/TAX cells through mutual regulation with CTSL by inhibiting EMT. In the same context, our findings suggested that miRNA-200c might be a potential treatment for non-small lung cancer. In our previously study, we have performed luciferase reporter assay to study whether CTSL is direct or indirect target of miRNA-200c. We selected the nucleotides 164 from exon 8 of the CTSL 3'UTR, but the results shown that in these sequences of CTSL, the luminescence intensity was not changed. It suggested that CTSL may be indirect target of miRNA-200c, there may be another regulatory factors, such as smad2, snail, p300, $\mathrm{MGMT}^{[52]}$. It is reported that miRNA-200c could regulate the expression of MGMT via c-Myb ${ }^{[52]}$, and in our laboratory, we also found the expression of MGMT in A549/TAX cells was higher than A549 cells, maybe the effect of miRNA-200c to CTSL is through regulating the expression of MGMT. Future studies should also investigate whether known miRNAs are differentially expressed in A549 and A549/TAX cells, or whether other miRNAs can suppress paclitaxel resistance induced by CTSL.

\section{Abbreviations}

CTSL, Cathepsin L; EMT, Epithelial-mesenchymal transition; PTX, Paclitaxel; ZEB1, Zinc Finger E-Box Binding Homeobox 1; ZEB2, Zinc Finger E-Box Binding Homeobox 2; A549/TAX, Paclitaxel-resistant A549 cells.

\section{Acknowledgements}

This work was supported by grants from the National Natural Science Foundation of China (Grant No 81773768, 81072656 and 81373430).

\section{Author contribution}

Zhong-qin LIANG and Yi-fan ZHAO designed the research; Yi-fan ZHAO, Mei-ling HAN, Ya-jie XIONG, and Long WANG conducted experiments; Yi-fan ZHAO, Yao FEI, Xiao SHEN, and Ying ZHU contributed new reagents or analytic tools; Yi-fan ZHAO and Mei-ling HAN performed data analysis; and Yi-fan ZHAO wrote the manuscript.

\section{References}

1 Kallianos A, Tsimpoukis S, Zarogoulidis P, et al. Measurement of exhaled alveolar nitrogen oxide in patients with lung cancer: a friend from the past still precious today. Oncol Targets Ther 2013; 6: 60913.

2 Maslyar DJ, Jahan TM, Jablons DM. Mechanisms of and potential treatment strategies for metastatic disease in non-small cell lung cancer. Semin Thorac Cardiovasc Surg 2004; 16: 40-50.

3 Smith W, Khuri FR. The care of the lung cancer patient in the 21st century: a new age. Semin Oncol 2004; 31: 11-5.

4 Greenberger LM, Lothstein L, Williams SS, Horwitz SB. Distinct P-glycoprotein precursors are overproduced in independently isolated drug-resistant cell lines. Proc Natl Acad Sci U S A 1988; 85: 3762-6.

5 Bylaite M, Moussali H, Marciukaitiene I, Ruzicka T, Walz M. Expression of cathepsin $\mathrm{L}$ and its inhibitor hurpin in inflammatory and neoplastic skin diseases. Exp Dermatol 2006; 15: 110-8.

6 Taqi C, Gover S, Dhanial J, Goyal S, Goyal M, Grover A. Mechanistic insights into mode of action of novel natural cathepsin $L$ inhibitors. BMC Gennomics 2013; 14: S10.

7 Tholen M, Wlianski J, Stolze B, Chiabudini M, Gajda M, Bronsert P, et al. Stress-resistant translation of cathepsin L mRNA in breast cancer progression. J Biol Chem 2015; 290: 15758-69.

8 Fei $\mathrm{Y}$, Xiong $\mathrm{Y}$, Zhao $\mathrm{Y}$, Wang $\mathrm{W}$, Han M, Wang L, et al. Cathepsin $L$ knockdown enhances curcumin-mediated inhibition of growth, migration, and invasion of glioma cells. Brain Res 2016; 1646: 5808.

9 Zheng X, Chu PM, Brikin BL, Rebbaa A. Senescence-initiated reversal of drug resistance:specific role of cathepsin L. Cancer Res 2004; 64: 1773-80.

10 Zheng X, Chu F, Chou P, Gallati C, Dier U, Mirkin BL, et al. Cathepsin $L$ inhibition suppresses drug resistance in vitro and in vivo: a putative mechanism. Am J Physiol Cell Physiol 2009; 296: C65-74.

11 Zhang Q, Han M, Wang W, Song Y, Chen G, Wang Z, et al. Downregulation of cathepsin $L$ suppresses cancer invasion and migration by inhibiting transforming growth factor- $\beta$-mediated epithelial-mesenchymal transition. Oncol Rep 2015; 33: 1851-9. 
12 Han ML, Zhao YF, Tan CH, Xiong YJ, Wang WJ, Wu F, et al. Cathepsin $\mathrm{L}$ upregulation-induced EMT phenotype is associated with the acquisition of cisplatin or paclitaxel resistance in A549 cells. Acta Pharmacol Sin 2016; 37: 1606-22.

13 Sui H, Shi C, Yan Z, Wu M. Overexpression of Cathepsin L is associated with chemoresistance and invasion of epithelial ovarian cancer. Oncotarget 2016; 7: 45995-6001.

14 Cui F, Wang W, Wu D, He X, Wu J, Wang M. Overexpression of Cathepsin $L$ is associated with gefiinib resistance in non-small lung cancer. Clin Trans Oncol 2016; 18: 722-7.

15 L'Espérance S, Popa I, Bachvarova M, Plante M, Patten N, Wu L, et al. Gene expression profiling of paired ovarian tumors obtained prior to and following adjuvant chemotherapy: molecular signatures of chemoresistant tumors. Int J Oncol 2006; 29: 5-24.

16 Frankel LB, Lund AH. MicroRNA regulation of autophagy. Carcinogenesis 2012; 33: 2018-25.

17 Brozovic A, Duran GE, Wang YC, Francisco EB, Sikic BI. Molecular oncology the miRNA-200 family differentially regulates sensitivity to paclitaxel and carboplatin in human ovarian carcinoma OVCAR-3 and MES-OV cells. Mol Oncol 2015; 9: 1678-93.

18 Castilla MA, Moreno-Bueno G, Romero-Perez L, Van De Vijver K, Biscuola M, Lopez-Garcia MA, et al. Micro-RNA signature of the epithelial-mesenchymal transition in endometrial carcinosarcoma. J Pathol 2011; 223: 72-80.

19 Thiery JP. Epithelial-mesenchymal transitions in tumour progression. Nat Rev Cancer 2002; 2: 442-54.

20 De Craene B, Berx G. Regulatory networks defining EMT during cancer initiation and progression. Nat Rev Cancer 2013; 13: 97-110.

21 Cochrane DR, Spoelstra NS, Howe EN, Nordeen SK, Richer JK. MicroRNA-200c mitigates invasiveness and restores sensitivity to microtubule-targeting chemotherapeutic agents. Mol Cancer Ther 2009; 8: 1055-66.

22 Li Y, VandenBoom TG 2nd, Kong D, Wang Z, Ali S, Philip PA, et al. Up-regulation of miR-200 and let-7 by natural agents leads to the reversal of epithelial-tomesenchymal transition in gemcitabineresistant pancreatic cancer cells. Cancer Res 2009; 69: 6704-12.

23 Ceppi P, Mudduluru G, Kumarswamy R, Rapa I, Scagliotti GV, Papotti M, et al. Loss of miR-200c expression induces an aggressive, invasive, and chemoresistant phenotype in non-small cell lung cancer. Mol Cancer Res 2010; 8: 1207-16.

24 Mateescu B, Batista L, Cardon M, Gruosso T, de Feraudy Y, Mariani 0 , et al. miR-141 and miR-200a act on ovarian tumorigenesis by controlling oxidative stress response. Nat Med 2011; 17: 1627-35.

25 Cittelly DM, Dimitrova I, Howe EN, Cochrane DR, Jean A, Spoelstra NS, et al. Restoration of miR-200c to ovarian cancer reduces tumor burden and increases sensitivity to paclitaxel. Mol Cancer Ther 2012; 11: 2556-65.

26 Fu J, Rodova M, Nanta R, Meeker D, Van Veldhuizen PJ, Srivastava RK, et al. NPV-LDE-225 (Erismodegib) inhibits epithelial mesenchymal transition and self-renewal of glioblastoma initiating cells by regulating miR-21, miR-128, and miR-200. Neurol Oncol 2013; 15: 691-706.

27 Prislei S, Martinelli E, Mariani M, Raspaglio G, Sieber S, Ferrandina G, et al. MiR-200c and HuR in ovarian cancer. BMC Cancer 2013; 13: 72.

28 Park SM, Gaur AB, Lengyel E, Peter ME. The miR-200 family determines the epithelial phenotype of cancer cells by targeting the E-cadherin repressors ZEB1 and ZEB2. Genes Dev 2008; 22: 894907.

29 Puisieux A, Brabletz T, Caramel J. Oncogenic roles of EMT inducing transcription factors. Nat Cell Biol 2014; 16: 488-94.

30 Chen HB, Zheng HT. MiRNA-200c represses migration and invasion of gastic cancer SGC-7901 cells by inhibiting expression of fibronectin 1. Eur Rev Med Pharmacol 2017; 21: 1753-8.

31 Lee H, Kim C, Kang H, Tak H, Ahn S, Yoon SK, et al. MiRNA-200a$3 p$ increases 5 -fluorourail resistance by regulating dual specificity phosphatase 6 expression open. Exp Mol Med 2017; 49: e327.

32 Bae SM, Lim W, Jeong W, Kim J, Bazer FW, Song G. Expression and regulation of avian cathepsin $\mathrm{L}$ in the oviduct during molting. Gen Comp Endocrinol 2014; 204: 88-94.

33 Liu S, Tetzlaff MT, Cui R, Xu X. miRNA-200c inhibits melanoma progression and drug resistance through down-regulation of Bmi-1. Am J Pathol 2012; 181: 1823-35.

34 Kim JJ, Yin B, Christudass CS, Terada N, Rajagopalan K, Fabry B, et al. Acquisition of paclitaxel resistance is associated with a more aggressive and invasive phenotype in prostate cancer. J Cell Biochem 2013; 114: 1286-93.

35 Byles V, Zhu L, Lovaas JD, Chmilewski LK, Wang J, Faller DV, et al. SIRT1 induces EMT by cooperating with EMT transcription factors and enhances prostate cancer cell migration and metastasis. Oncogene 2012; 31: 4619-29.

36 Zhong X, Zheng L, Shen J, Zhang D, Xiong M, Zhang Y, et al. Suppression of MicroRNA 200 family expression by oncogenic KRAS activation promotes cell survival and epithelial-mesenchymal transition in KRAS-driven cancer. Mol Cell Biol 2016; 36: 2742-54.

37 Jedeszko C, Sloane BF. Cysteine cathepsin in human cancer. Biolchem 2004; 385: 1017-27.

38 Zhang H, Zhang L, Wei L, Gao X, Tang LI, Gong W, et al. Knockdown of cathepsin $L$ sensitizes ovarian cancer cells to chemotherapy. Oncol Lett 2016; 11: 4235-9.

39 Wu Y, Xiao Y, Ding X, Zhuo Y, Ren P, Zhou C, et al. A miR-200b/200c/429binding site polymorphism in the 3 ' untranslated region of AP- $2 \alpha$ gene is associated with cisplatin resistance. PLoS One 2011; 6: e29043.

40 Adam L, Zhong M, Choi W, Qi W, Nicoloso M, Arora A, et al. miR-200 expression regulates epithelial-to-mesenchy-mal transition in bladder cancer cells and reverses resistance to epidermal growth factor receptor therapy. Clin Cancer Res 2009; 15: 5060-72.

41 Chang L, Guo F, Wang Y, Lv Y, Huo B, Wang L, Liu W. MicroRNA-200c regulates the sensitivity of chemotherapy of gastric cancer SGC7901/ DDP cells by directly targeting RhoE. Pathol Oncol Res 2014; 20 : 93-8.

42 Ma C, Huang T, Ding YC, Yu W, Wang Q, Meng B, et al. MicroRNA200c overexpression inhibits chemoresistance, invasion and colony formation of human pancreatic cancer stem cells. Int J Clin Exp Pathol 2015; 8: 6533-9.

43 Housman G, Byler S, Heerboth S, Lapinska K, Longacre M, Snyder N, et al. Drug resistance in cancer: an overview. Cancers (Basel) 2014; 6: 1769-92.

44 Shang Y, Cai X, Fan D. Roles of epithelial-mesenchymal transition in cancer drug resistance. Curr Cancer Drug Targets 2013; 13: 915-29.

45 Singh A, Settleman J. EMT, cancer stem cells and drug resistance: An emerging axis of evil in the war on cancer. Oncogene 2010; 29 : 4741-51.

46 Lamouille S, Xu J, Derynck R. Molecular mechanisms of epithelialmesenchymal transition. Nat Rev Mol Cell Biol 2014; 15: 178-96.

47 FarizNurwidya, et al. Epithelial mesenchymal transition in drug reistance and metastasis of lung cancer. Cancer Res Treat 2012; 44: 151-6.

48 Pan Q, Meng L, Ye J, Wei X, Shang Y, Tian Y, et al. Transcriptional repression of miR-200c family members by Nanog in colon cancer cells induces epithelial-mesenchymal transition (EMT). Cancer Lett 2017; 392: 26-38.

49 Ahn SM, Cha JY, Kim J, Kim D, Trang HTH, Kim YM, et al. Smad3 
regulates E-cadherin via miRNA-200 pathway. Oncogene 2012; 31: 3051-9.

50 Li J, Yuan J, Yuan X, Zhao J, Zhang Z, Weng L, et al. MicroRNA-200b inhibits the growth and metastasis of glioma cells via targeting ZEB2. J Mol Med 2016; 48: 541-50.

51 Korpal M, Lee ES, Hu G, Kang Y. The miR-200 family inhibits epithelial-mesenchymal transition and cancer cell migration by direct targeting of E-cadherin transcriptional repressors ZEB1 and ZEB2. J Biol Chem 2008; 283: 14910-4.

52 Perdigão-Henriques R, Petrocca F, Altschuler G, Thomas MP, Le MTN, Tan SM, et al. miR-200 promotes the mesenchymal to epithelial transition by suppressing multiple members of the Zeb2 and Snail1 transcriptional repressor complexes. Oncogene 2016; 35: 158-72. 